\title{
Phytosterol Accumulation Results in Ventricular Arrhythmia, Impaired Cardiac Function and Death
}

Hongfei Ge

Amgen Research

Gongxin Liu

Amgen Research

Tracy M. Yamawaki

Amgen Research

Caroline Tao

Amgen Research

Shawn T. Alexander

Amgen Research

Kimberly Ly

Amgen Research

Preston Fordstrom

Amgen Research

Artem A. Shkumatov

Amgen Research

Chi-Ming Li

Amgen Research

Sridharan Rajamani

Amgen Research

Mingyue Zhou

Amgen Research

Brandon Ason ( $\sim$ bason@amgen.com )

Amgen Research

\section{Research Article}

Keywords: Heart failure (HF), pathological conditions, accelerate disease pathogenesis, premature ventricular contractions

Posted Date: May 10th, 2021

DOl: https://doi.org/10.21203/rs.3.rs-474251/v1 
License: (c) (i) This work is licensed under a Creative Commons Attribution 4.0 International License. Read Full License 
Phytosterol accumulation results in ventricular arrhythmia, impaired cardiac function and death

Hongfei Ge ${ }^{1 *}$, Gongxin Liu ${ }^{1 *}$, Tracy M. Yamawaki ${ }^{2}$, Caroline Tao ${ }^{1}$, Shawn T. Alexander ${ }^{1}$, Kimberly Ly, Preston Fordstrom ${ }^{1}$, Artem A. Shkumatov ${ }^{3}$, Chi-Ming Li ${ }^{2}$, Sridharan Rajamani ${ }^{3}$, Mingyue Zhou ${ }^{1}$ and Brandon Ason ${ }^{1 *}$

${ }^{1}$ Cardiometabolic Disorders Therapeutic Area, Amgen Research, South San Francisco, CA, USA

${ }^{2}$ Genomic Analysis Unit, Amgen Research, South San Francisco, CA, USA

${ }^{3}$ Translational Safety \& Bioanalytical Sciences, Amgen Research, South San Francisco, CA, USA

*equal contribution

**Correspondance

Brandon Ason

bason@amgen.com

Amgen, Inc

1120 Veterans Blvd

South San Francisco, CA

94080 


\section{ABSTRACT}

Heart failure (HF) and cardiac arrhythmias share overlapping pathological mechanisms that act cooperatively to accelerate disease pathogenesis. Cardiac fibrosis is associated with both pathological conditions. Our previous work identified a link between phytosterol accumulation and cardiac injury in a mouse model of phytosterolemia, a rare disorder characterized by elevated circulating phytosterols and increased cardiovascular disease risk. Here, we uncover a previously unknown pathological link between phytosterols and cardiac arrhythmias in the same animal model. Phytosterolemia resulted in inflammatory pathway induction, premature ventricular contractions (PVC) and ventricular tachycardia (VT). Both pharmacological and genetic inhibition of phytosterol absorption prevented the induction of both pathways. Inhibition of phytosterol absorption reduced inflammation and cardiac fibrosis, improved cardiac function, reduced the incidence of arrhythmias and increased survival in a mouse model of phytosterolemia. Collectively, this work identified a pathological mechanism whereby elevated phytosterols result in inflammation and cardiac fibrosis leading to impaired cardiac function, arrhythmias and sudden death. These phytosterolemia-associated comorbidities provide novel insight into the underlying pathophysiological mechanism that predispose these patients to increased risk of sudden cardiac death. 


\section{INTRODUCTION}

Heart failure (HF) remains an egregious disease with poor prognosis that is estimated to afflict $\sim 64$ million people worldwide ${ }^{1}$. Patients with HF are predisposed to developing cardiac arrhythmias including complex ventricular arrhythmias, ventricular tachycardia and atrial fibrillation ${ }^{2,3}$. Likewise, cardiac arrhythmias predispose individuals to HF and contribute to worsening HF prognosis. This relationship between HF and cardiac arrhythmias is driven by numerous shared co-morbidities and overlapping pathologies.

Cardiac fibrosis is strongly considered as both a cause and a consequence of arrhythmias and HF 4-6. Cardiac fibrosis can manifest in patients with rare inflammatory diseases such as sarcoidosis resulting in congestive HF, cardiac arrhythmias, or both ${ }^{7}$. The severity of cardiac fibrosis correlates with the incidence and progression of both $\mathrm{HF}$ and arrhythmias and increases the risk of sudden cardiac death ${ }^{5,8}$. We recently demonstrated that phytosterolemia can cause cardiac fibrosis in mice and was associated with premature death.

Phytosterolemia is a rare disorder characterized by elevated phytosterols in the blood and a remarkably high risk of cardiovascular disease ${ }^{9-12}$. Phytosterols are solely acquired from dietary sources such as vegetable oil, soybeans, nuts and seeds. Despite being present at comparable quantities with cholesterol in a typical human diet, phytosterols are largely prevented from entering circulation as they are effectively excreted through bile via the sterol transporter heterodimer, ATP-binding cassette sub-family G member 5/8 (ABCG5/8) ${ }^{13-15}$. This is essential for human health as rare ABCG5/8 loss of function variants lead to phytosterolemia and increase the risk of developing coronary artery disease as well as the risk of experiencing a myocardial infarction or sudden cardiac death ${ }^{4,16-22}$. 
Niemann-Pick C1-Like 1 (NPC1L1) is the opposing sterol transporter ${ }^{23-25}$. While ABCG5/8 is responsible for dietary sterol excretion, NPC1L1 is responsible for the absorption of dietary cholesterol and phytosterols. NPC1L1 and ABCG5/8 together control dietary sterol input to maintain cholesterol and phytosterol homeostasis. The NPC1L1 inhibitor, ezetimibe, reduces both dietary cholesterol and phytosterol absorption and is recommended for patients with phytosterolemia. Both ezetimibe and genetic inactivation of NPC1L1 can block intestinal absorption of phytosterols and prevent their accumulation within the blood and liver of ABCG5/8 deficient mice.

Here, we show that genetic inactivation of NPC1L1 compensated for the loss of ABCG5/8 function by preventing phytosterol induced inflammation and cardiac fibrosis. This led to improved cardiac function, ablated arrhythmias and increased survival. Bulk RNA sequencing of cardiac tissue revealed that phytosterol accumulation was associated with an induction of inflammatory response genes as well as genes associated with arrhythmias. These diseases-associated changes in gene expression were absent in ezetimibe treated mice, concurrent genetic inactivation of NPC1L1, or both. We additionally show that phytosterolemia induced by genetic inactivation of ABCG5/8 led to both premature ventricular contractions (PVC) and ventricular tachycardia (VT), while ezetimibe treatment reduced the incidence of these arrhythmias. Taken together, our findings represent a novel, previously unknown link between phytosterolemia, arrhythmias and sudden cardiac death. 


\section{RESULTS}

Genetic inactivation of NPC1L1 corrects phytosterolemia induced inflammation, cardiac fibrosis, cardiac dysfunction and the incidence of death caused by ABCG5/8 deficiency - Our previous work demonstrated that phytosterolemia induced by feeding ABCG5/8 double knockout (DKO) mice a phytosterol enriched diet led to cardiac fibrosis and death ${ }^{26}$. It also revealed that three weeks of ezetimibe treatment reduced both plasma phytosterols and associated cardiac fibrosis ${ }^{26}$. Here, we utilized NPC1L1 knockout (KO) mice to determine if reducing phytosterol absorption by genetic inactivation could relieve phytosterolemia associated pathologies, including mortality ${ }^{27}$. Mice that were heterozygous for the ABCG5/8 deletion (DKO) were crossed to heterozygous NPC1L1 KO mice to produce 1) wild type, 2) NPC1L1 KOs, 3) ABCG5/8 DKOs, and 4) NPC1L1, ABCG5/8 triple knockout (TKO) littermates. The animals produced from this cross were between $99-100 \%$ congenic to the C57B1/6J background line. Twenty-week old littermates were placed on the phytosterol enriched diet (chow supplemented with $0.2 \%$ stigmasterol, $0.2 \%$ sitosterol) for 7 weeks. Sterol concentrations were determined by LC-MS/MS

to differentiate cholesterol from the individual phytosterols. ABCG5/8 DKO mice fed a phytosterol enriched diet exhibited a significant increase in plasma phytosterols relative to wild type or NPC1L1 KO littermate controls (Fig. 1A-F). The NPC1L1, ABCG5/8 TKO mice exhibited a significant reduction in phytosterol levels relative to the ABCG5/8 DKO littermates. Interestingly, plasma $\beta$-sitosterol, stigmasterol, coprostanol and the coprostanol/cholesterol ratio in the NPC1L1, ABCG5/8 TKOs were significantly higher relative to the NPC1L1 KO and wild type littermates indicating NPC1L1 inactivation is unable to fully counteract ABCG5/8 deficiency. 
We next assessed cardiac function using echocardiography. Left ventricular function was compared between conscious littermates at baseline, 3- and 6-weeks following placement on the phytosterol enriched diet. Cardiac function appeared normal for ABCG5/8 DKO mice at baseline, declining at week 3 and worsening further 6 weeks following the switch to the phytosterol enriched diet as evident by a significant decrease in ejection fraction (EF), fractional shortening (FS) and an increase in end systolic volume (ESV) and end diastolic volume (EDV) (Fig. 1G-J). This was associated with a modest decrease in stroke volume (SV) and heart rate (HR), reaching significance at week 6 for the ABCG5/8 DKOs compared to littermates (Fig. 1K-L). There was no difference in the heart to brain weight ratios between groups after 7 weeks indicating that LV dilation was not associated with a change in heart mass (Supplemental Fig. 1). The phytosterol induced decline in cardiac function was prevented through the simultaneous inactivation of NPC1L1 and ABCG5/8 as the TKO exhibited measures of cardiac function that were comparable to both wild type and NPC1L1 KO littermate controls.

Histopathological assessment of hearts after 7 weeks phytosterol diet-challenge revealed that NPC1L1, ABCG5/8 TKOs exhibited significantly lower cardiac fibrosis relative to ABCG5/8 DKO littermates. Fibrotic scores from H\&E and trichrome stained hearts were comparable for the NPC1L1, ABCG5/8 TKOs when compared to either NPC1L1 KOs or wild type littermates. This contrasts with the ABCG5/8 DKO group, which exhibited a fibrotic score of 4 on a $0-4$ point scale indicating that the hearts in this group contained diffusely distributed fibrosis in the left and right ventricles as well as the interventricular septum, which separated and often compressed individual cardiomyocytes. Fibrotic areas contained mature and immature collagen with active fibroblasts mixed with inflammatory cells (macrophages, lymphoid cells, and rarely neutrophils) indicating an actively ongoing fibrotic process. (Fig. 1M-N). The degree of cardiac fibrosis correlated with 
circulating inflammatory cytokine levels at week 3 (Fig. 10-Q). TNFa, TIMP-1, KC/GRO were all significantly elevated for the ABCG5/8 DKO group, consistent with phytosterolemia eliciting an inflammatory response triggering fibroblast activation and fibrosis.

Elevated circulating phytosterols, inflammatory cytokines and cardiac fibrosis was associated with an increased incidence of death for ABCG5/8 DKO mice, while the reduction in phytosterols, cytokines and cardiac fibrosis corresponded to a reduction in mortality for NPC1L1, ABCG5/8 TKO littermates (Fig. 1R). ABCG5/8 DKO mice began to die after 4 weeks of phytosterolenriched diet challenge with only 4 out of 12 mice (30\%) remaining after 7 weeks. This was preceded by a decline in body weight for the ABCG5/8 DKO group (Supplemental Fig. 2). Collectively, these data indicate that NPC1L1 inactivation compensates for the loss of ABCG5/8 by attenuating phytosterol accumulation thereby reducing inflammation, cardiac fibrosis and mortality.

The NPC1L1 inhibitor, ezetimibe, only partially inhibits phytosterol transport - To determine if the NPC1L1 inhibitor, ezetimibe, is sufficient to completely inhibit phytosterol transport, we compared plasma phytosterol levels in NPC1L1 KO mice to mice treated with a maximum efficacious dose of ezetimibe $(10 \mathrm{mg} / \mathrm{kg}$, QD) for 3 weeks. Three weeks of daily ezetimibe administration resulted in a significant reduction in plasma phytosterols and a significant increase plasma cholesterol levels (Fig. 2A-D). Genetic inactivation of NPC1L1 resulted in a greater reduction in circulating phytosterols relative to ezetimibe treatment as evident by the significantly lower plasma phytosterol levels in NPC1L1, ABCG5/8 TKOs relative to ABCG5/8 DKO littermates treated with ezetimibe. Reducing plasma phytosterol levels either by genetic inactivation of NPC1L1 or through NPC1L1 inhibition with ezetimibe led to a significant reduction in cardiac fibrosis (Fig. 2E-F). 


\section{Phytosterolemia led to inflammation and arrhythmia pathway induction - To explore}

phytosterolemia induced changes in gene expression, we next examined the gene expression profiles from 20 cardiac tissue samples comprising ABCG5/8 DKO and ABCG5/8, NPC1L1 TKO mice fed a phytosterol-enriched diet and administered ezetimibe (10 mg/kg, QD) or vehicle control. To explore the similarity in gene expression across conditions, we performed principal component analysis (PCA). We observed separation of cardiac tissue isolated from ezetimibe and vehicle treated DKO groups in the first principle component $(\mathrm{PC} 1)$, which explained 25.6\% of the variance. Additionally, DKO ezetimibe treated cardiac samples clustered with TKO ezetimibe and vehicle treated cardiac tissue samples (Fig. 3A).

We next identified differentially expressed (DE) genes in DKO vehicle treated samples relative to DKO ezetimibe treated and TKO cardiac tissue samples. DEseq2 analysis using a cutoff of padj $<0.01$ uncovered 5,548 DE genes in DKO ezetimibe treatment and 5,166 DE genes in the TKO relative to vehicle treated DKO (Fig. 3B, Supplemental Table 1). DE genes from the two analyses demonstrated high overlap with 1,565 shared genes significantly upregulated and 2,562 genes downregulated in both DKO ezetimibe treated and TKO vehicle treated samples. Furthermore, we observed high consistency in the directionality of expression and few inverse effects in the two comparisons with only $3 \mathrm{DE}$ genes with significant upregulation in DKO ezetimibe treated or TKO samples and downregulated in the other comparison (Fig. 3C). Overall, the comparison between differentially expressed genes from the DKO vs. TKO-vehicle, DKO vs. DKO-ezetimibe and DKO vs. TKO-ezetimibe samples exhibited a high degree of overlap to one another (Fig. 3D).

With DE genes identified, we next explored functional pathways affected by ezetimibe treatment or NPC1L1 gene inactivation using gene ontology (GO) enrichment analysis and Ingenuity 
Pathway Analysis (IPA). We observed a high degree of overlap in GO terms enriched in the comparisons. Notably, many of the highly significant GO terms are related to immune function and inflammation such as GO:0002376, immune system process [p < 1e-30 both comparisons] and GO:0006954, inflammatory response [p $<1 \mathrm{e}-30$ both comparisons] (Fig. 4A, Supplemental Table 1). Taking a closer look at candidate inflammation-related genes, we observed upregulation of these genes in the vehicle treated DKO cardiac tissue samples relative to ezetimibe treated DKO and both ezetimibe and vehicle treated TKO cardiac tissue samples (Fig. 4B). Similarly, IPA analysis identified significant enrichment of inflammatory response pathways [p $=5.89 \mathrm{e}-84$ (DKO v TKO), 5.48e-83 (DKO vs treated)] and gene expression of DE genes in this category also showed upregulation in DKO vehicle treated samples relative to ezetimibe and vehicle treated TKO samples (Fig. 4C). Additionally, in the IPA analysis, the arrhythmia disease category demonstrated significant enrichment [p = 1.58e-6 (DKO v TKO), 3.69e-6 (DKO vs treated)] with similar gene expression patterns of DE genes in ezetimibe treated DKO and TKO samples (Fig. 4D).

Phytosterols and cardiac arrhythmia - We hypothesized that increased ventricular arrythmias may be the cause of death in both patients and ABCG5/8 DKO mice with phytosterolemia. We evaluated the hypothesis by analyzing ABCG5/8 DKO mice, as this model recapitulates the sudden death observed in patients with phytosterolemia. ECG recordings were analyzed from 22-26 week-old ABCG5/8 DKO mice and wild type littermates that were fed a phytosterol enriched diet at baseline and after 4 weeks of treatment with either a maximum efficacious dose of ezetimibe $(10 \mathrm{mg} / \mathrm{kg}, \mathrm{QD})$ or vehicle control. Wild type littermate mice were free of ventricular arrhythmias both at baseline and at 4 weeks post-treatment. In contrast, ABCG5/8 DKO mice exhibited a higher incidence of premature ventricular contractions (PVC), over 80\%, and ventricular 
tachycardia (VT), over 55\%, at baseline (Fig. 5A-B). The incidence of PVCs and VTs in DKO mice trended higher in the vehicle treated ABCG5/8 DKO mice at the week 4 time point. In contrast, ezetimibe treated mice exhibited a slight decrease in PVCs (90\% vs 66\%) but a significant reduction in VTs (80\% vs 33\%) when compared to DKO vehicle treated mice (Fig. 5C).

\section{DISCUSSION}

In our current work, we extend our previous study which revealed that phytosterolemia could lead to cardiac fibrosis resulting in premature death (10). Here, we show that phytosterolemia led to an increase in incidence of ventricular arrhythmias. Genetic inactivation of NPC1L1 or inhibition with ezetimibe reduced blood phytosterols, inflammation, cardiac fibrosis, ventricular arrhythmias and sudden cardiac death. Through the identification of several novel phytosterolemia associated comorbidities, we now have a better understanding of the underlying pathophysiology that links phytosterolemia to increased mortality. We have shown that the accumulation of phytosterols results in chronic inflammation, cardiac fibrosis, cardiac injury, arrhythmia and death. Our data support a model where phytosterols accumulate in circulation triggering inflammation that results in fibroblast activation. This activation leads to structural rearrangements to the heart causing cardiac injury and sudden cardiac death (Fig. 6).

The high incidence of PVCs and VTs is the result of fibrosis induced changes in the molecular architecture of cardiac tissue where individual cells were frequently observed to be separated and compressed. The normal electrical connectivity of cardiac tissue is disrupted and the vulnerability to arrhythmias is increased ${ }^{28-30}$. These anatomical and functional changes have been shown to alter normal cardiac electrical conduction pathways and may explain why phytosterolemia patients are at increased risk of experiencing a myocardial infarction or sudden cardiac death as arrhythmia is often attributed to be the underlying cause ${ }^{31-34}$. 
Phytosterolemia caused by deleterious mutations within the ABCG5/8 heterodimeric transporter is estimated to affect more than 1 in 200,000 individuals within the general population ${ }^{31}$. Patients with phytosterolemia exhibit elevated circulating phytosterols. Additionally, phytosterol deposition in tissues can reach more than one hundred times normal levels in these individuals. They are also at increased risk of developing cardiovascular disease.

Both phytosterolemia and hypercholesterolemia are commonly described as cardiovascular disorders that are generally assumed to lead to cardiovascular disease through similar pathological mechanisms. While cardiovascular disease is typically driven by hypercholesterolemia induced vascular atherosclerosis, our work from this study uncovered a novel and distinct pathological mechanism for phytosterolemia induced cardiovascular related death that is driven by cardiac fibrosis and associated arrhythmias.

Phytosterolemia patients are sometimes misdiagnosed with severe hypercholesterolemia, as standard analytical methods used to quantify cholesterol are unable to distinguish cholesterol from structurally related phytosterols ${ }^{26}$. This would help to explain why phytosterolemia patients respond poorly to statin therapy, as statins, which inhibit cholesterol synthesis and do not lower circulating phytosterols, which are solely acquired by dietary absorption ${ }^{32,35,36}$.

Our data indicate that blocking phytosterol absorption through NPC1L1 inhibition can reduce inflammation, cardiac fibrosis, ventricular arrhythmia and death in mice. Given the fact that individuals with phytosterolemia are at risk of being misdiagnosed with hypercholesterolemia, our work underscores the importance of raising awareness of this disorder ${ }^{37,38}$. This is critically important, considering the issues surrounding misdiagnosis, the fact that statins are the first line standard of care therapy for hypercholesterolemia and are known to provide limited clinical benefit for phytosterolemia patients relative to the NPC1L1 inhibitor, ezetimibe ${ }^{31,39,40}$. 
Our work additionally reveals that a maximum efficacious dose of ezetimibe does not completely inhibit NPC1L1 mediated phytosterol transport in mice with phytosterolemia. The reduced but significantly elevated phytosterols following a maximum efficacious dose of ezetimibe may lead to a more gradual development of cardiac fibrosis that was not detectable within the duration of our studies. Given that NPC1L1 genetic inactivation resulted in even lower phytosterol levels raises the possibility that a more potent inhibitor of NPC1L1 mediated phytosterol transport could lower phytosterol levels further and be a more efficacious therapy to treat phytosterolemia. It also raises the possibility that targeting ABCG5/8 with an agonist may represent a particularly valuable therapeutic strategy for phytosterolemia patients.

\section{MATERIALS AND METHODS}

In Vivo - All studies reported in this manuscript follow the recommendations in the ARRIVE guidelines (https://arriveguidelines.org). All rodent studies were conducted at Amgen Inc and were approved by the Amgen Institutional Animal Care and Use Committee (IACUC) and complied with the Final Rules of the Animal Welfare Act regulations (Code of Federal Regulations, Title 9), the Public Health Service Policy on Humane Care and Use of Laboratory Animals in the Office of Laboratory Animal Welfare (2002), and the Guide for the Care and Use of Laboratory Animals from the National Research Council (1996). Mice were housed in ventilated cages on corncob bedding with 12:12 hour light:dark cycle. C57B/6-NPC1L1 KO and C57B/6ABCG5/ABCG8 DKO (B6;129S6-Del(17Abc5-Abc8)1Hobb/J) mice were licensed from Mount

Sinai and UT Southwestern, respectively ${ }^{27,41}$. Both lines were backcrossed to C57Bl/6 mice obtained from Charles River to achieve 99-100\% homozygosity, respectively. The NPC1L1, ABCG5/8 TKOs were generated by crossing heterozygous NPC1L1 KOs to heterozygous ABCG5/8 DKOs. Mice had ad libitum access food and water. For ezetimibe administration, 
vehicle or $10 \mathrm{mg} / \mathrm{kg}$ ezetimibe (Fisher, 50-753-2773, Cat\#163222-33-1) were administered daily via oral gavage for 3 weeks. Mice were euthanized and tissues were excised and fixed in $10 \%$ buffered formalin overnight and then transferred to $70 \%$ ethanol.

Echocardiography - Non-invasive echocardiograms were obtained on conscious mice using a Vevo 2100 imaging system. Hair was removed using commercially available depilatory cream the day before, mice were placed on a stage and sonography gel was applied on the thorax. Twodimensional targeted 'M-mode' imaging was obtained from the short-axis view at the level of the papillary muscle. Animals were subsequently wiped clean of sonography gel, and returned to their home cage.

Electrocardiogram Recording - The mice were induced with 3\% isoflurane and maintained with $1-1.5 \%$ isoflurane delivered with COMPAC5 vaporizer. Loss of the righting limb reflex by applying a strong toe pinch was used to judge the anesthetic depth of isoflurane before starting the procedure. Mice were then placed in a dorsal recumbence position on a heated pad. The limbs were fixed with tape. After ECG recording, mouse was allowed time to wake up and was then returned to its cage.

Six lead (I, II, III, aVF, aVL, aVR) electrocardiogram (ECG) was recorded using a digital acquisition system (Biopac, MP160) from subdermal ECG needle electrodes (29 gauge, $12 \mathrm{~mm}$ long; AD Instruments) inserted subcutaneously into the left and right forelimb and into left hindlimb. The surface ECG tracings were filtered using a high pass setting of $1 \mathrm{~Hz}$ and low pass setting of $150 \mathrm{~Hz}$. The signal was acquired for 5 minutes using AcqKnowledge 5 software.

ECG was manually analyzed offline. Arrhythmic events, specifically, premature ventricular contraction (PVC) and ventricular tachycardia (VT) were carefully examined to estimate the 
arrhythmia incidence. PVC was count in when occurs $>1$ per minute. Consecutive 3 ventricular beating was counted as VT.

LC-MS/MS - Plasma samples were analyzed using liquid chromatography with tandem mass spectrometry (LC-MS/MS, performed by Metabolon Inc., Durham, NC) for the contents of the following sterols (after hydrolysis of sterol esters): cholesterol, $\beta$-sitosterol, campesterol, and stigmasterol. Samples analyses were carried out in a 96-well plate format containing two calibration curves and six QC samples (per plate) to monitor method performance. The mass spectrometer was operated in selected ion monitoring (SIM) mode. The masses of the extracted ions were 458.4 (cholesterol), 382.4 (campesterol), 396.4 ( $\beta$-sitosterol), and 523.2 (stigmasterol). Histology - Following paraffin embedding, tissue sections were stained with hematoxylin and eosin (H\&E) (Biocare IPCS5006G20), and trichrome using standard protocols. Images were obtained using Aperio Digital Pathology Slide Scanner (Leica Biosystems) and Aperio ImageScope v12 software. The evaluation of slides was done by board-certified veterinary pathologist based on following scoring criteria: $0=$ no fibrosis, $1-$ minimal fibrosis (barely noticeable interstitial or focal replacement fibrosis), 2 - mild fibrosis (easily detectable multifocal interstitial or replacement fibrosis) , 3 - moderate fibrosis (consistently observed in all chambers and interconnected lattice of fibrosis), 4 - severe fibrosis (same as 3 with large fibrotic areas of replaced cardiac tissue).

Circulating Cytokines - Serum TNF-alpha, KC/GRO and TIMP-1 concentrations were measured at week 3 using the MSD V-PLEX Cytokine Panel 1 Mouse Kit (K15245D) and the R\&D Systems Mouse TIMP1 Quantikine ELISA Kit (MTM100), respectively. These assays were performed as described within the product protocols. 
Bulk RNA Seq and associated analyses - $150 \mathrm{ng}$ of total RNA with ERCC spike-ins was used as input for the TruSeq Stranded mRNA Library Prep (Illumina). Libraries were sequenced on an Illumina HiSeq4000 to a minimum depth of 30 million 150x150 paired-end reads. Alignment and quantification were performed using Oshell v10.0.1.111 ${ }^{42,43}$. Reads were aligned to the OmicsoftGencode.V19 mouse reference based on GRCm38. Quantile normalization of FPKM values was performed using the 70th percentile of gene expression according to the following formula: FPKQ=10 FPKM/(FPKM 70th Percentile) ${ }^{44}$.

Principal component analysis (PCA) was performed using the prcomp function in R on centered and scaled log transformed FPKQ data of genes detected in at least one sample. This analysis identified a single outlier (triple knock-out no treatment sample) and was removed from downstream analyses. DEseq2 v1.26.0 was used to identify differentially expressed (DE) genes between double knock-out untreated vs treated or double knock-out vs triple knockout using count data and the Wald test to determine $\mathrm{p}$ values ${ }^{45}$. Gene Ontology enrichment analysis was performed on DE gene analysis $\mathrm{p}$ values using the topGO package v2.31.1 ${ }^{46}$. Further functional analysis of DE genes was performed using IPA (QIAGEN Inc., https://www.qiagenbioinformatics.com/products/ingenuity-pathway-analysis; ${ }^{47}$ ).

Quantification and statistical analysis - All data are reported as the mean \pm S.E.M. The differences between the mean values were tested for statistical significance by one-way ANOVA as indicated in figure legends. 
Data Availability - All data generated and analyzed during this study are included in this article and supplementary information files. Sequencing data has been deposited in GEO under the accession GSE173123. Additional data and information about this study are available from the corresponding author on request. Further information and requests for resources and reagents should be directed to the lead contact, Dr. Brandon Ason (bason@amgen.com).

\section{AUTHOR CONTRIBUTIONS}

HG, GL, TMY, CT, STA, KL, PF, AAS, CML, SR, MZ and BA made substantial contributions to either the experimental design, data acquisition, analysis, or interpretation of the results. BA drafted the initial version of the manuscript. HG, GL, TMY, CT, STA, KL, PF, AAS, CML, SR and MZ made edits to the final version of the manuscript. All authors approved the submitted version of the manuscript.

\section{ACKNOWLEDGEMENTS}

We thank Richard Haven, Nilo Allotta and Danielle Pruitt for technical assistance. C.T. was supported by the Amgen Postdoctoral Fellowship program.

\section{ADDITIONAL INFORMATION}

All authors were employed by Amgen at the time the work was conducted, and all work was funded by Amgen, Inc. 


\section{REFERENCES}

1 Groenewegen, A., Rutten, F. H., Mosterd, A. \& Hoes, A. W. Epidemiology of heart failure. Eur J Heart Fail 22, 1342-1356 (2020).

2 Podrid, P. J., Fogel, R. I. \& Fuchs, T. T. Ventricular arrhythmia in congestive heart failure. Am J Cardiol 69, 82G-95G; discussion 95G-96G, doi:10.1016/00029149(92)91257-5 (1992).

3 Alvarez, C. K., Cronin, E., Baker, W. L. \& Kluger, J. Heart failure as a substrate and trigger for ventricular tachycardia. J Interv Card Electrophysiol 56, 229-247, doi:10.1007/s10840-019-00623-x (2019).

4 Frangogiannis, N. G. Cardiac fibrosis: Cell biological mechanisms, molecular pathways and therapeutic opportunities. Mol Aspects Med 65, 70-99, doi:10.1016/j.mam.2018.07.001 (2019).

5 González, A., Schelbert, E. B., Díez, J. \& Butler, J. Myocardial Interstitial Fibrosis in Heart Failure: Biological and Translational Perspectives. J Am Coll Cardiol 71, 16961706, doi:10.1016/j.jacc.2018.02.021 (2018).

6 de Jong, S., van Veen, T. A., van Rijen, H. V. \& de Bakker, J. M. Fibrosis and cardiac arrhythmias. J Cardiovasc Pharmacol 57, 630-638, doi:10.1097/FJC.0b013e318207a35f (2011).

7 Markatis, E., Afthinos, A., Antonakis, E. \& Papanikolaou, I. C. Cardiac sarcoidosis: diagnosis and management. Rev Cardiovasc Med 21, 321-338, doi:10.31083/j.rcm.2020.03.102 (2020).

8 Aoki, T. et al. Prognostic impact of myocardial interstitial fibrosis in non-ischemic heart failure. -Comparison between preserved and reduced ejection fraction heart failure. Circ $J$ 75, 2605-2613, doi:10.1253/circj.cj-11-0568 (2011).

9 Sudhop, T. \& von Bergmann, K. Sitosterolemia—a rare disease. Z Kardiol 93, 921-928, doi:10.1007/s00392-004-0165-2 (2004).

10 Salen, G. et al. Lethal atherosclerosis associated with abnormal plasma and tissue sterol composition in sitosterolemia with xanthomatosis. Journal of Lipid Research 26, 11261133 (1985).

11 Teupser, D. et al. Genetic Regulation of Serum Phytosterol Levels and Risk of Coronary Artery Disease. Circulation: Cardiovascular Genetics 3, 331-339, doi:10.1161/circgenetics.109.907873 (2010).

12 Consortium, T. I. B. C. K. C. A. D. Large-Scale Gene-Centric Analysis Identifies Novel Variants for Coronary Artery Disease. PLOS Genetics 7, e1002260, doi:10.1371/journal.pgen.1002260 (2011).

13 Kuksis, A., Marai, L., Myher, J. J. \& Geher, K. Identification of plant sterols in plasma and red blood cells of man and experimental animals. Lipids 11, 581-586, doi:10.1007/bf02532869 (1976).

14 von Bergmann, K., Sudhop, T. \& Lütjohann, D. Cholesterol and Plant Sterol Absorption: Recent Insights. The American Journal of Cardiology 96, 10-14, doi:http://dx.doi.org/10.1016/j.amjcard.2005.03.014 (2005).

15 Lammert, F. \& Wang, D. Q. New insights into the genetic regulation of intestinal cholesterol absorption. Gastroenterology 129, 718-734, doi:10.1016/j.gastro.2004.11.017 (2005). 
16 Helgadottir, A. et al. Genetic variability in the absorption of dietary sterols affects the risk of coronary artery disease. Eur Heart J 41, 2618-2628 (2020).

17 Kawamura, R., Saiki, H., Tada, H. \& Hata, A. Acute myocardial infarction in a 25-yearold woman with sitosterolemia. J Clin Lipidol 12, 246-249, doi:10.1016/j.jacl.2017.10.017 (2018).

18 Nomura, A. et al. Heterozygous ABCG5 Gene Deficiency and Risk of Coronary Artery Disease. Circ Genom Precis Med 13, 417-423, doi:10.1161/circgen.119.002871 (2020).

19 Teupser, D. et al. Genetic regulation of serum phytosterol levels and risk of coronary artery disease. Circ Cardiovasc Genet 3, 331-339, doi:10.1161/circgenetics.109.907873 (2010).

20 Stender, S., Frikke-Schmidt, R., Nordestgaard, B. G. \& Tybjærg-Hansen, A. The ABCG5/8 cholesterol transporter and myocardial infarction versus gallstone disease. $J$ Am Coll Cardiol 63, 2121-2128, doi:10.1016/j.jacc.2013.12.055 (2014).

21 Aulchenko, Y. S. et al. Loci influencing lipid levels and coronary heart disease risk in 16 European population cohorts. Nat Genet 41, 47-55 (2009).

22 Koeijvoets, K. C. et al. ABCG8 gene polymorphisms, plasma cholesterol concentrations, and risk of cardiovascular disease in familial hypercholesterolemia. Atherosclerosis 204, 453-458, doi:10.1016/j.atherosclerosis.2008.09.018 (2009).

23 Altmann, S. W. et al. Niemann-Pick C1 Like 1 protein is critical for intestinal cholesterol absorption. Science. 303, 1201-1204. doi: 1210.1126/science.1093131. (2004).

24 Garcia-Calvo, M. et al. The target of ezetimibe is Niemann-Pick C1-Like 1 (NPC1L1). Proc Natl Acad Sci U S A. 102, 8132-8137. doi: 8110.1073/pnas.0500269102. Epub 0500262005 May 0500269131. (2005).

25 Brown, J. M. \& Yu, L. Opposing Gatekeepers of Apical Sterol Transport: Niemann-Pick C1-Like 1 (NPC1L1) and ATP-Binding Cassette Transporters G5 and G8 (ABCG5/ABCG8). Immunol Endocr Metab Agents Med Chem. 9, 18-29. doi: 10.2174/187152209788009797. (2009).

26 Tao, C., Shkumatov, A. A., Alexander, S. T., Ason, B. L. \& Zhou, M. Stigmasterol accumulation causes cardiac injury and promotes mortality. Commun Biol. 2:20., 10.1038/s42003-42018-40245-x. eCollection 42019. (2019).

27 Davies, J. P., Scott, C., Oishi, K., Liapis, A. \& Ioannou, Y. A. Inactivation of NPC1L1 causes multiple lipid transport defects and protects against diet-induced hypercholesterolemia. J Biol Chem. 280, 12710-12720. doi: 12710.11074/jbc.M409110200. Epub 409112005 Jan 409110225. (2005).

28 Disertori, M., Masè, M. \& Ravelli, F. Myocardial fibrosis predicts ventricular tachyarrhythmias. Trends Cardiovasc Med 27, 363-372, doi:10.1016/j.tcm.2017.01.011 (2017).

29 Nguyen, T. P., Qu, Z. \& Weiss, J. N. Cardiac fibrosis and arrhythmogenesis: the road to repair is paved with perils. J Mol Cell Cardiol 70, 83-91 (2014).

30 Centurión, O. A. et al. Myocardial Fibrosis as a Pathway of Prediction of Ventricular Arrhythmias and Sudden Cardiac Death in Patients With Nonischemic Dilated Cardiomyopathy. Crit Pathw Cardiol 18, 89-97, doi:10.1097/hpc.0000000000000171 (2019).

31 Tada, H. et al. Sitosterolemia, Hypercholesterolemia, and Coronary Artery Disease. $J$ Atheroscler Thromb. 25, 783-789. doi: 710.5551/jat.RV17024. Epub 12018 Jul 17020. (2018). 
32 Kawamura, R., Saiki, H., Tada, H. \& Hata, A. Acute myocardial infarction in a 25-yearold woman with sitosterolemia. J Clin Lipidol. 12, 246-249. doi: 210.1016/j.jacl.2017.1010.1017. Epub 2017 Nov 1020. (2018).

33 Ludhwani, D., Goyal, A. \& Jagtap, M. in StatPearls (@) 2021, StatPearls Publishing LLC., 2021).

34 Schwartz, P. J. et al. Inherited cardiac arrhythmias. Nat Rev Dis Primers 6, 58 (2020).

35 Nguyen, L. B. et al. Regulation of cholesterol biosynthesis in sitosterolemia: effects of lovastatin, cholestyramine, and dietary sterol restriction. J Lipid Res. 32, 1941-1948. (1991).

36 von Bergmann, K., Sudhop, T. \& Lutjohann, D. Cholesterol and plant sterol absorption: recent insights. Am J Cardiol. 96, 10D-14D. doi: 10.1016/j.amjcard.2005.1003.1014. (2005).

37 Wang, W. et al. A case of sitosterolemia misdiagnosed as familial hypercholesterolemia: A 4-year follow-up. J Clin Lipidol 12, 236-239, doi:10.1016/j.jacl.2017.10.008 (2018).

38 Lee, J. H. et al. High prevalence of increased sitosterol levels in hypercholesterolemic children suggest underestimation of sitosterolemia incidence. PLoS One 15, e0238079 (2020).

39 Tsubakio-Yamamoto, K. et al. Current therapy for patients with sitosterolemia--effect of ezetimibe on plant sterol metabolism. J Atheroscler Thromb. 17, 891-900. doi: 810.5551/jat.4614. Epub 2010 Jun 5511. (2010).

40 Salen, G. et al. Ezetimibe effectively reduces plasma plant sterols in patients with sitosterolemia. Circulation. 109, 966-971. doi: 910.1161/1101.CIR.0000116766.0000131036.0000116703. Epub 0000112004 Feb 0000116769. (2004).

$41 \mathrm{Yu}, \mathrm{L}$. et al. Disruption of Abcg5 and Abcg8 in mice reveals their crucial role in biliary cholesterol secretion. Proc Natl Acad Sci U S A. 99, 16237-16242. doi: 16210.11073/pnas.252582399. Epub 252582002 Nov 252582320. (2002).

42 Hu, J., Ge, H., Newman, M. \& Liu, K. OSA: a fast and accurate alignment tool for RNASeq. Bioinformatics 28, 1933-1934, doi:10.1093/bioinformatics/bts294 (2012).

43 Li, J., Hu, J., Newman, M., Liu, K. \& Ge, H. RNA-Seq Analysis Pipeline Based on Oshell Environment. IEEE/ACM Trans Comput Biol Bioinform 11, 973-978, doi:10.1109/tcbb.2014.2321156 (2014).

44 Robinson, M. D. \& Oshlack, A. A scaling normalization method for differential expression analysis of RNA-seq data. Genome Biol 11, R25 (2010).

45 Love, M. I., Huber, W. \& Anders, S. Moderated estimation of fold change and dispersion for RNA-seq data with DESeq2. Genome Biol 15, 550 (2014).

46 Alexa, A., Rahnenführer, J. \& Lengauer, T. Improved scoring of functional groups from gene expression data by decorrelating GO graph structure. Bioinformatics 22, 1600-1607, doi:10.1093/bioinformatics/btl140 (2006).

47 Krämer, A., Green, J., Pollard, J., Jr. \& Tugendreich, S. Causal analysis approaches in Ingenuity Pathway Analysis. Bioinformatics 30, 523-530 (2014). 
Fig. 1

A.

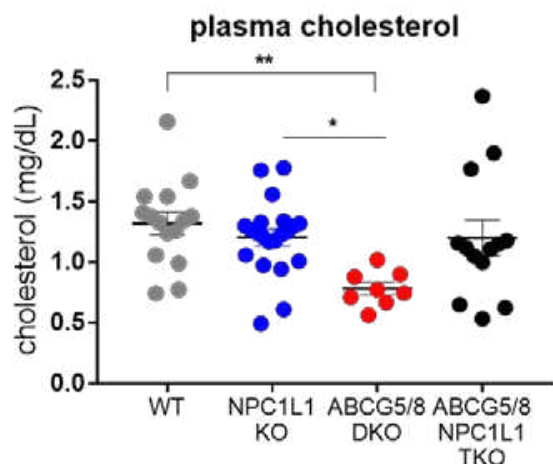

C.

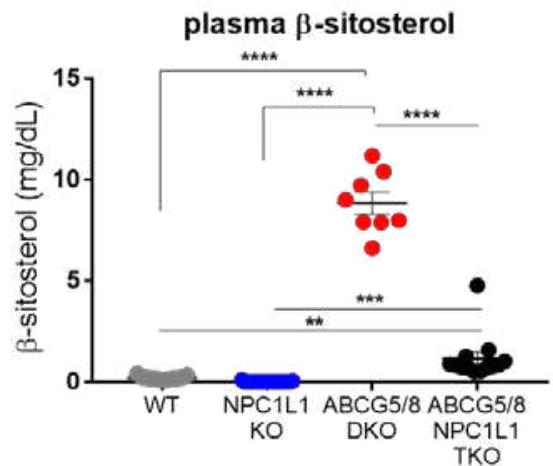

E.

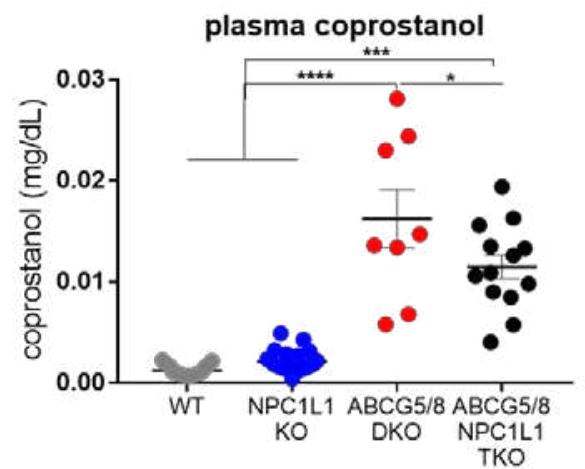

B.
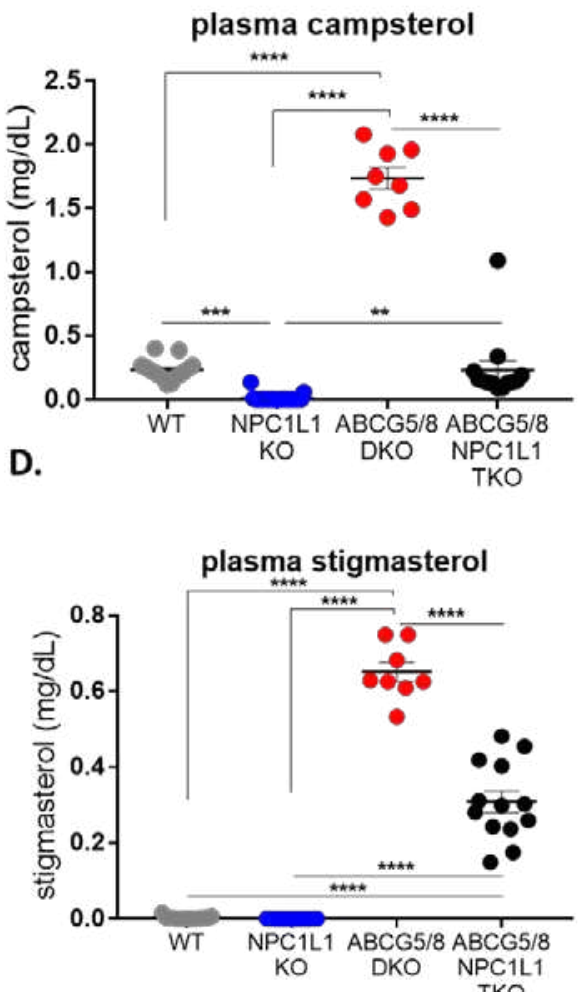

F.

coprostanol/cholesterol

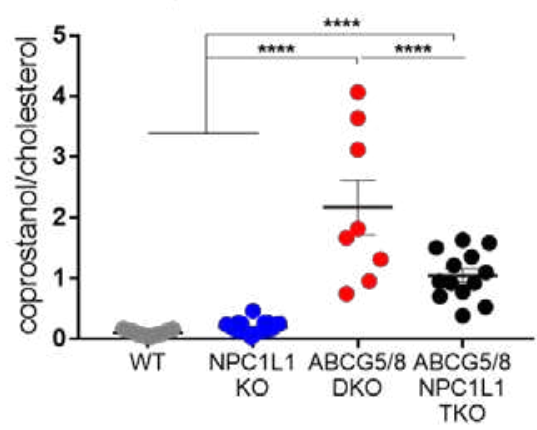


Fig. 1 cont

G.

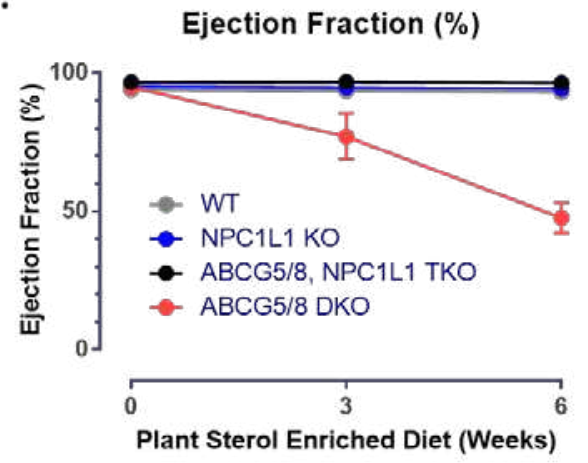

I.

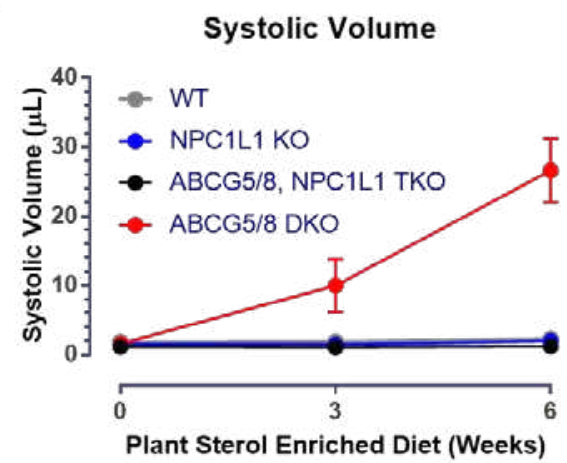

K.

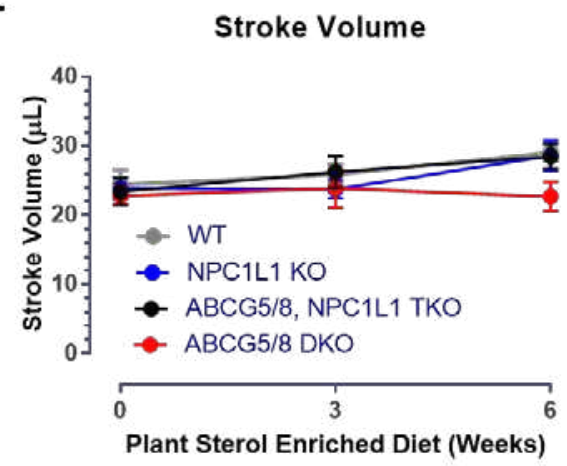

H.

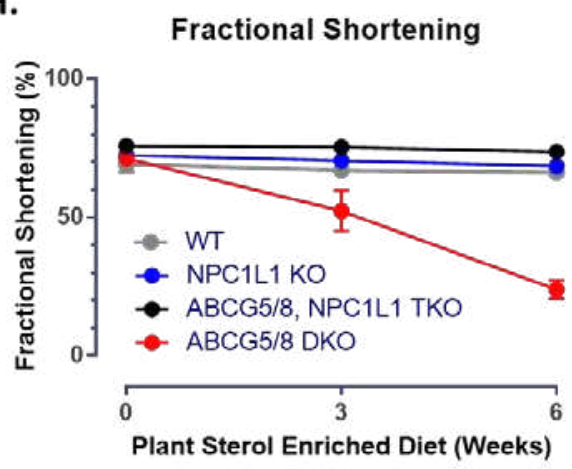

J.

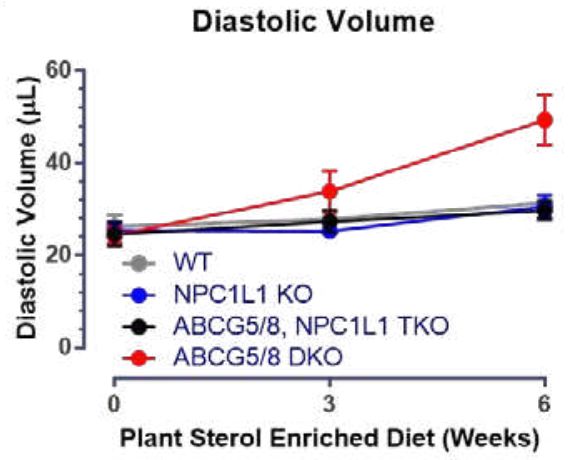

L.

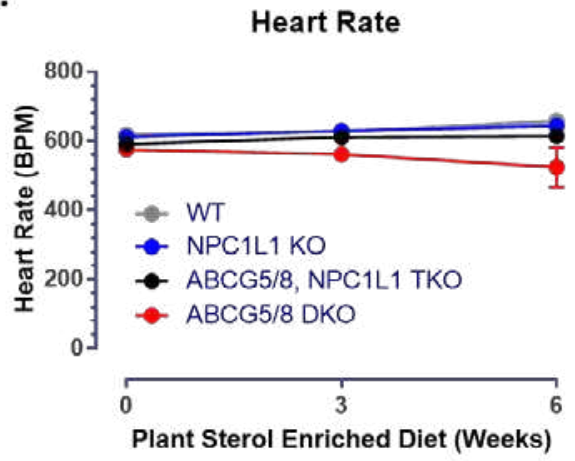


Fig. 1 cont

M.

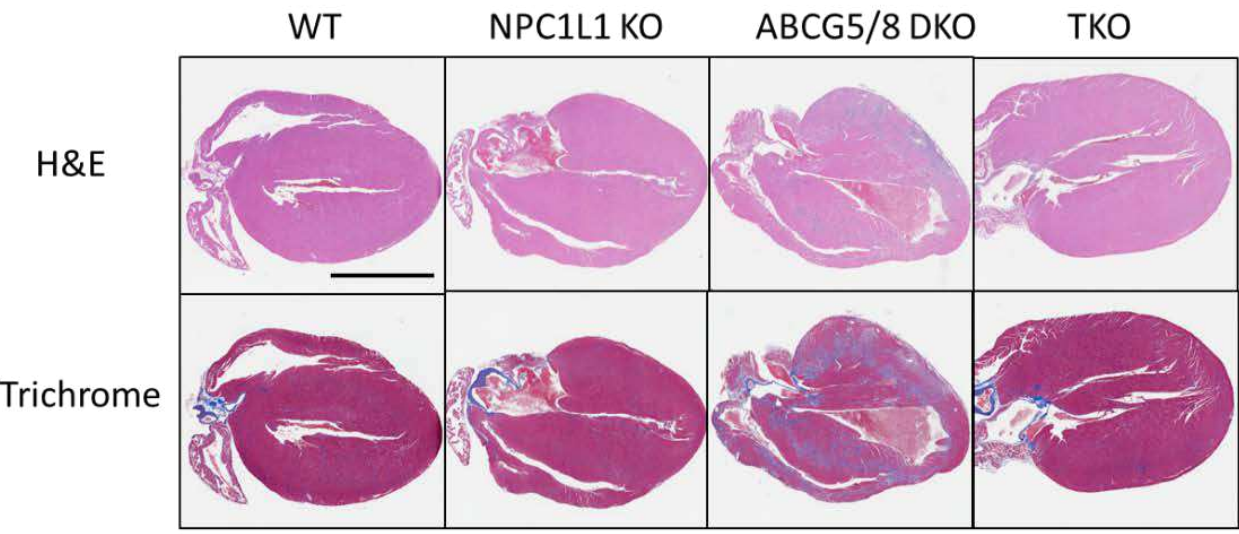

N.

Fibrosis Score (week 7)

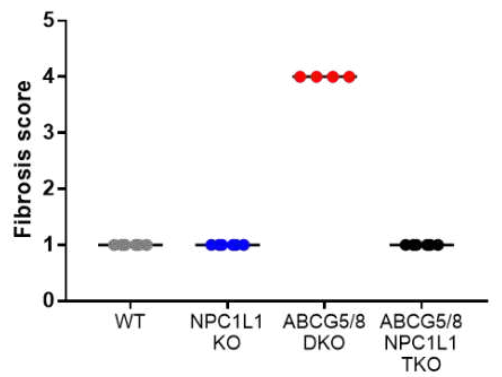

0.

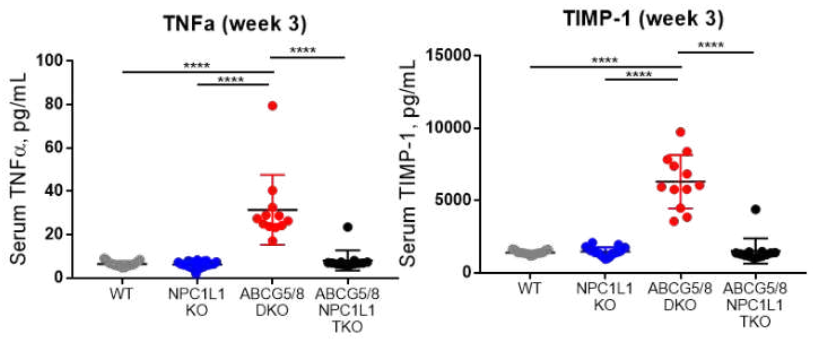

Q.

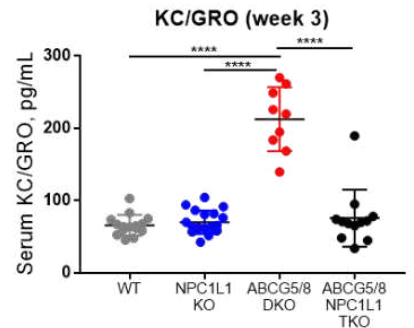




\section{Fig. 1 cont}

R.

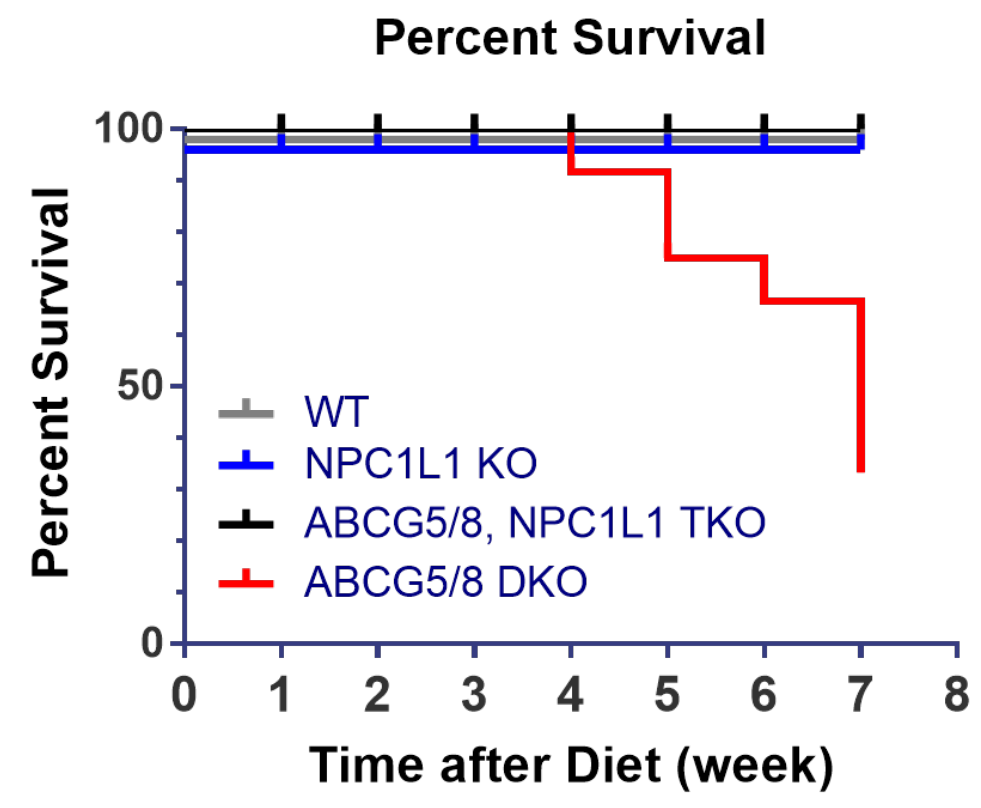

Fig. 1. The NPC1L1 / ABCG5/8 TKO exhibits less phytosterolemia relative to ABCG5/8

DKO littermates. The ABCG5/8 DKO led to a decrease of plasma cholesterol (A) and increase of four phytosterols (campsterol (B), $\beta$-sitosterol (C), stigmasterol (D), coprostanol (E)) as well as the coprostanol/cholesterol ratio $(\mathbf{F})$. Less but still significant levels of phytosterols accumulated in the TKO mice in comparison to the mouse cohorts carrying functional ABCG5/8 transporter. DKO - ABCG5/8 double knockout, TKO - ABCG5/8, NPC1L1 triple knockout. Individual data points (circles) are shown together with the sample mean (bar) \pm SEM. ABCG5/8 DKO mice exhibit decreased ejection fraction $(\mathbf{G})$ and fractional shortening $(\mathbf{H})$ and an increase in both systolic (I) and diastolic volume (J) with negligible changes to stroke volume (K) or heart rate $(\mathbf{L})$ indicative of impaired cardiac function and left ventricular dilation. These impairments in cardiac function are not observed for the TKO, NPC1L1 KO or WT littermate 
controls. DKO - ABCG5/8 double knockout, TKO - ABCG5/8, NPC1L1 triple knockout. Data represented as group means (circles) \pm SEM. WT: $N=15$ / group; NPC1L1 KO: $N=20-21$ / group; TKO: $\mathrm{N}=11-13$ / group; DKO: $\mathrm{N}=6-12$ /group. Data analyses performed blinded. (M) Representative images from WT, NPC1L1 KO, ABCG5/8 DKO and NPC1L1 / ABCG5/8 TKO at 7 weeks following the switch to the phytosterol enriched diet are shown. Scale bar equal 4 mm. (N) Cardiac fibrosis was scored as 0-within normal limits, 1-minimal, 2-mild, 3-moderate, and 4-severe. Severe fibrosis in the ABCG5/8 DKO group at week 7 was associated with a significant increase in several inflammatory markers (serum TNF- $\alpha(\mathbf{O})$, TIMP-1 $(\mathbf{P})$ and KC/GRO (Q)) at week 3. DKO - ABCG5/8 double knockout, TKO - ABCG5/8, NPC1L1 triple knockout. Individual data points (circles) \pm SEM are shown. Significance measured by one-way ANOVA - Tukey post-test (NS - not significant; $* \mathrm{P}<0.05 ; * * \mathrm{P}<0.01$; *** $\mathrm{P}<0.001 ; * * * * \mathrm{P}$ < 0.0001). (R) ABCG5/8 DKO mice died beginning 4 weeks post-initiation of the phytosterol enriched diet. In contrast, the phytosterol enriched diet did not result in death in the TKO, NPC1L1 KO or WT littermate controls out to week 7 post-diet induction. DKO - ABCG5/8 double knockout, TKO - ABCG5/8, NPC1L1 triple knockout. WT: N = 15/ group; NPC1L1 KO: $\mathrm{N}=21$ / group; TKO: $\mathrm{N}=13$ / group; DKO: $\mathrm{N}=12$ /group when mice were switched to the phytosterol enriched diet. 
Fig. 2

A.

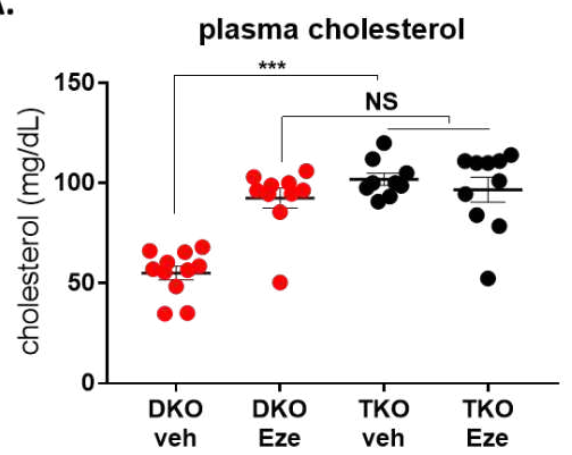

C.

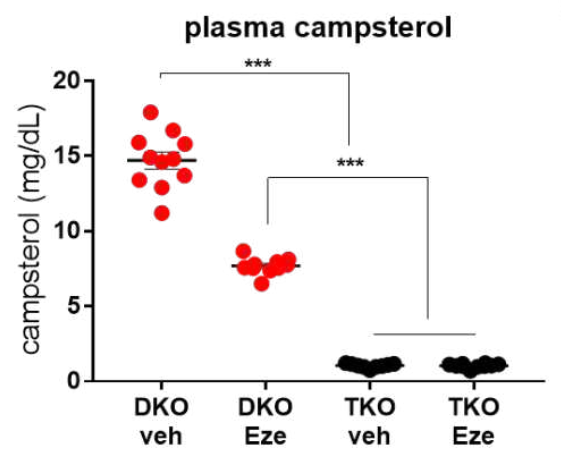

E.

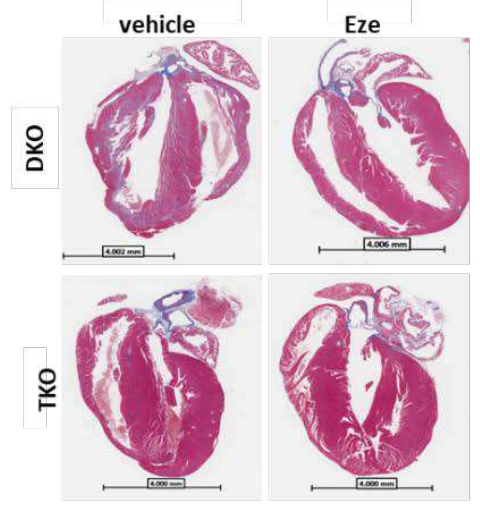

B.

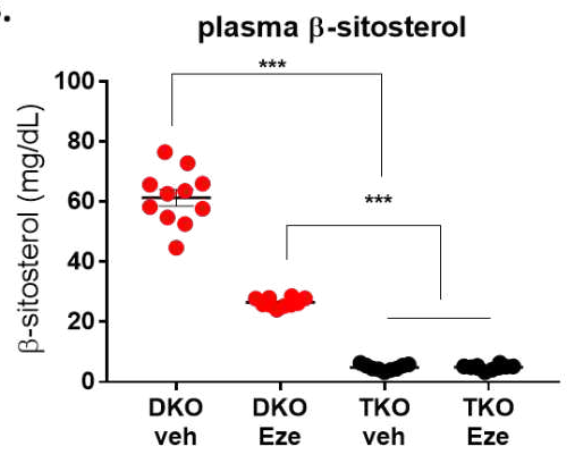

D.

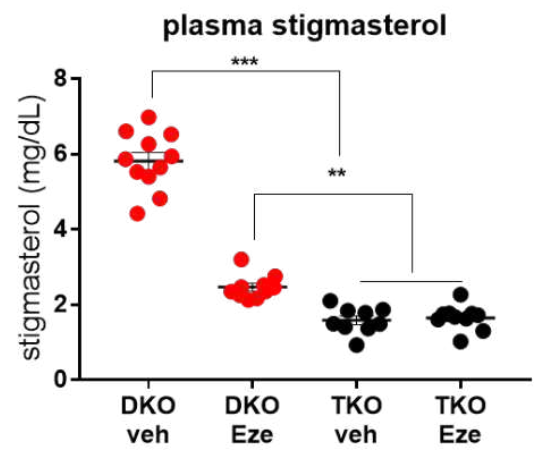

F.

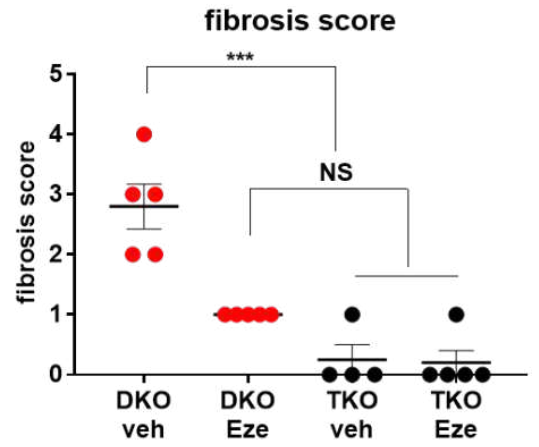

Fig. 2. The NPC1L1 inhibitor, ezetimibe, partially prevents ABCG5/8 deficiency-derived phytosterolemia. Ezetimibe treatment led to an increase of plasma cholesterol (A) and decreased of three phytosterols ( $\beta$-sitosterol $(\mathbf{B})$ campesterol $(\mathbf{C})$ and stigmasterol $(\mathbf{D}))$ in ABCG5/8 DKO mice. Plasma levels of phytosterols were lower in the NPC1L1 / ABCG5/8 TKO relative to the ABCG5/8 DKO littermates treated with ezetimibe. Both ezetimibe and NPC1L1 KO decreased 
ABCG5/8 DKO induce fibrosis (E, F). DKO - ABCG5/8 double knockout, TKO - ABCG5/8, NPC1L1 triple knockout, veh - vehicle, Eze - ezetimibe. Individual data points (circles) are shown together with the sample mean (bar) \pm SEM. Significance measured by one-way ANOVA - Tukey post-test (NS - not significant; $* \mathrm{P}<0.05$; ** $\mathrm{P}<0.01$; *** $\mathrm{P}<0.001$; **** $\mathrm{P}<0.0001)$. Histopathological assessment performed blinded. 
Fig. 3

A

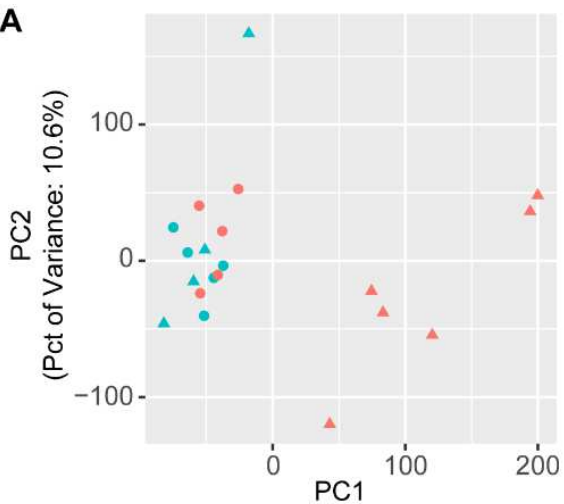

(Pct of Variance: $25.6 \%$ )

Genotype + Treatment

$\triangle A B C G 5 / 8$ DKO

- $A B C G 5 / 8$ DKO + Ezetimibe

$\triangle \mathrm{ABCG} 5 / 8 \mathrm{NPC} 1 \mathrm{~L} 1 \mathrm{TKO}$

- ABCG5/8 NPC1L1 TKO + Ezetimibe

C

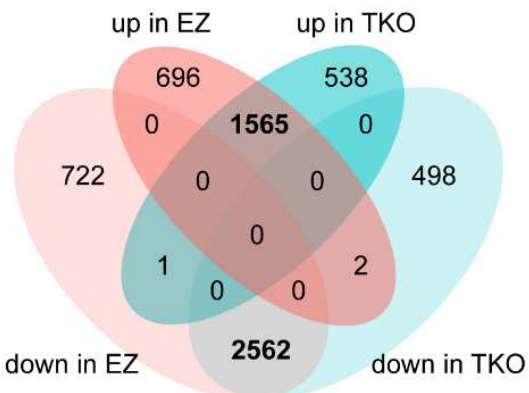

B

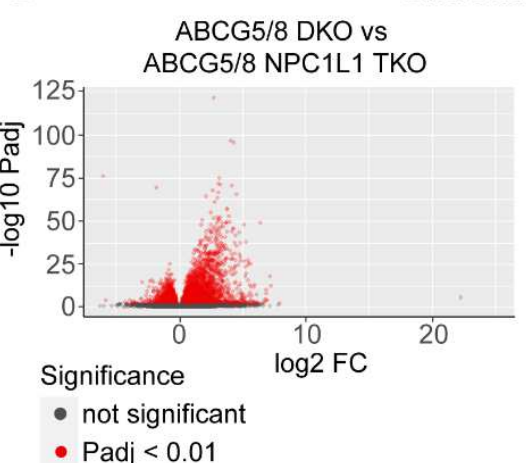

Differential Gene Expression

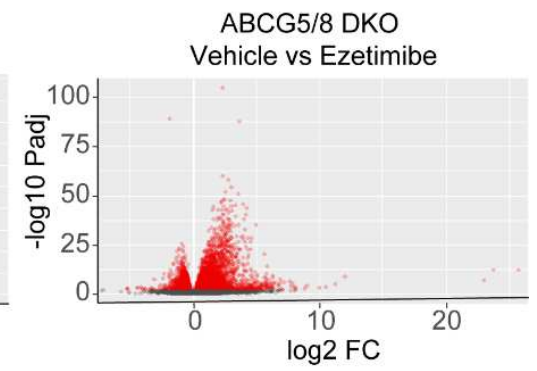

ABCG5/8 DKO

Vehicle vs Ezetimibe

D

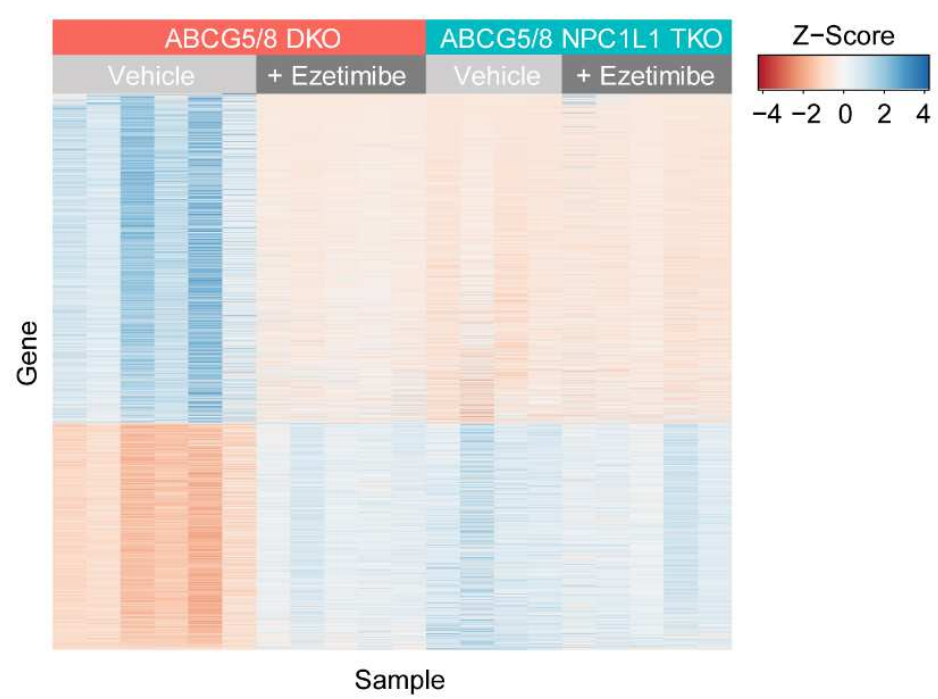

Fig. 3. RNA-seq analysis of heart samples. (A) Plot of first two components of principal component analysis (PCA) of gene expression. ABCG5/8 double knock-out (DKO) samples are filled with red and ABCG5/8 NPC1L1 triple knock-out (TKO) samples are filled with cyan. Vehicle samples are triangles and ezetimibe treated samples are circles. (B) Volcano plots of $\mathrm{p}$ values from differential expression analysis versus $\log 2$ fold change in gene expression as calculated using DEseq2 comparing DKO untreated to TKO or DKO ezetimibe treated samples. A positive fold change value indicates higher expression in ABCG5/8 DKO untreated samples.

(C) Venn diagram demonstrating overlap in differentially expressed (DE) genes from two 
comparisons. (D) Heatmap of gene expression (quantile normalized FPKM) Z-scores of DE genes identified comparing DKO and TKO conditions. 
Fig. 4

A

$$
\begin{array}{r}
\text { GO:0002376, immune system process } \\
\text { GO:0006952, defense response } \\
\text { GO:0006955, immune response } \\
\text { GO:0006954, inflammatory response } \\
\text { GO:0002682, regulation of immune system process } \\
\text { GO:0045087, innate immune response } \\
\text { GO:0006950, response to stress } \\
\text { GO:0009605, response to external stimulus } \\
\text { GO:0002252, immune effector process } \\
\text { GO:0001816, cytokine production } \\
\text { GO:0001775, cell activation } \\
\text { GO:0002684, positive regulation of immune system } \\
\text { GO:0050900, leukocyte migration } \\
\text { GO:0001817, regulation of cytokine production } \\
\text { GO:0044281, small molecule metabolic process } \\
\text { GO:0045321, leukocyte activation } \\
\text { GO:0009987, cellular process } \\
\text { G0:0009607, response to biotic stimulus } \\
\text { GO:0031347, regulation of defense response } \\
\text { GO:0043207, response to external biotic stimulus }
\end{array}
$$

\section{Comparison}

\section{- ABCG5/8 DKO vs ABCG5/8 NPC1L1 TKO}

- ABCG5/8 DKO vs ABCG5/8 DKO + Ezetimibe

C

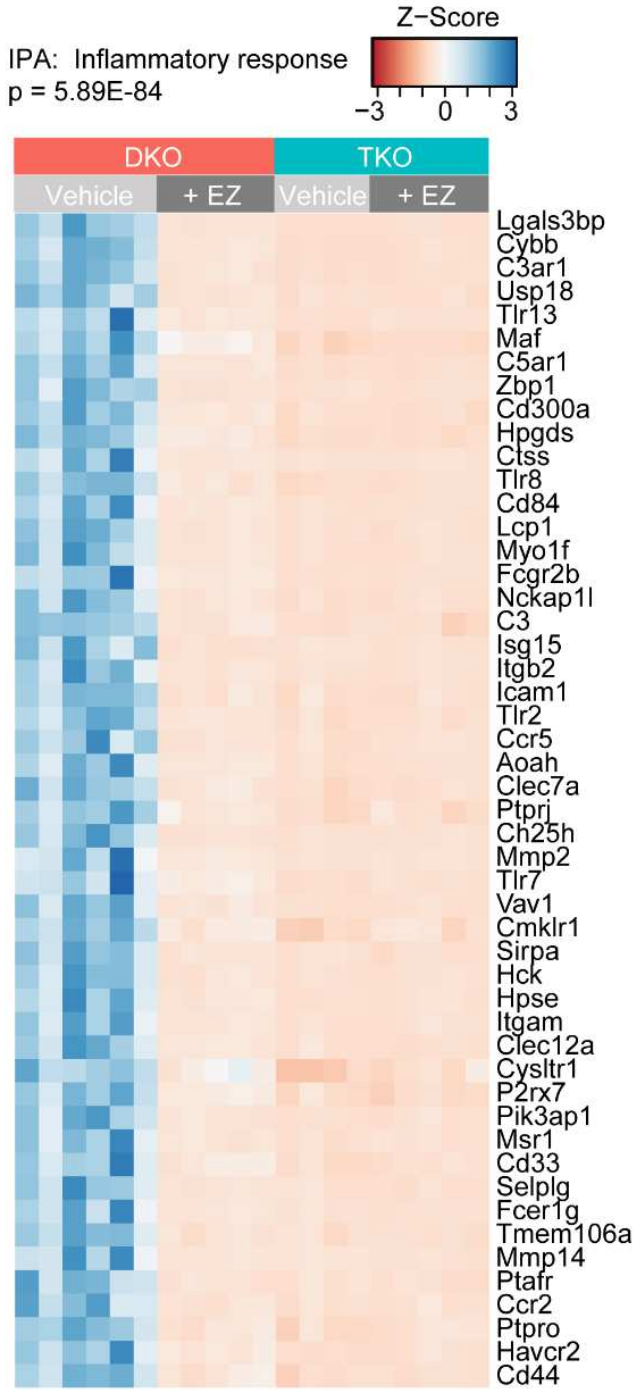

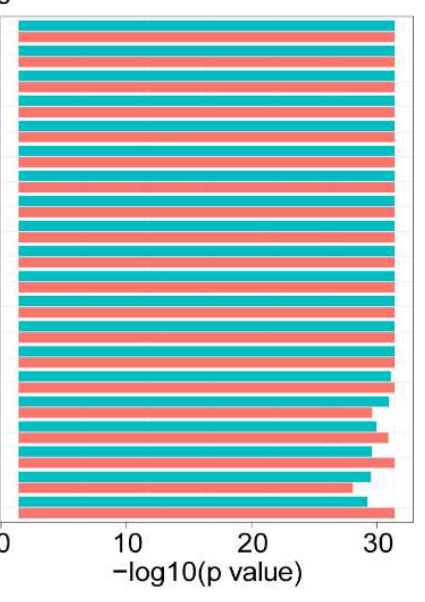

B

Inflammation Related Gene Expression

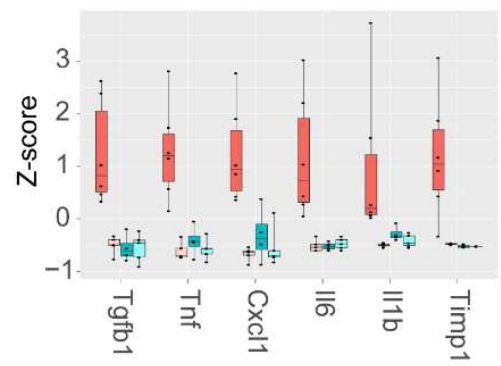

ABCG5/8 DKO

$\pm A B C G 5 / 8$ DKO + Ezetimibe

ABCG5/8 NPC1L1 TKO

ABCG5/8 NPC1L1 TKO + Ezetimibe

D

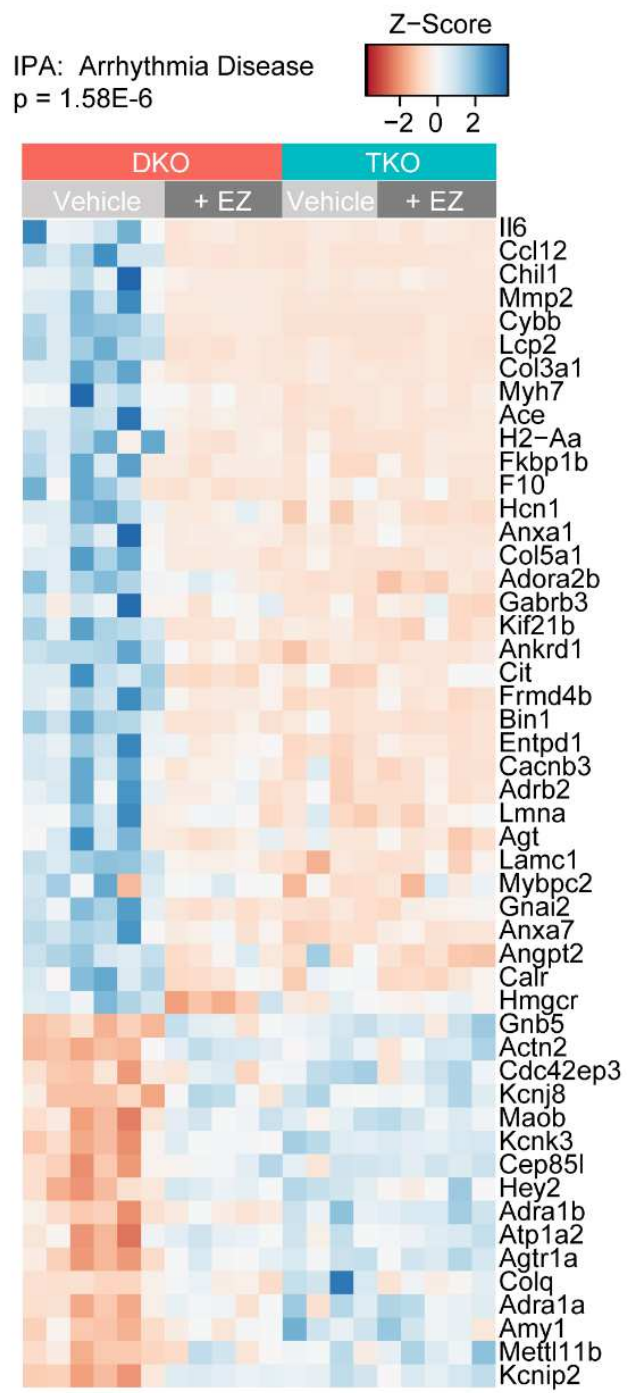


Fig. 4. Functional analysis of RNA-seq data. (A) Gene Ontology (GO) enrichment analysis of differentially expressed (DE) genes comparing ABCG5/8 double knock-out (DKO) samples to ABCG5/8 NPC1L1 triple knock-out (TKO) (cyan) or DKO ezetimibe (red) treated samples. P values for the top twenty GO categories from the DKO/TKO comparison are plotted. (B) Box plots of gene expression (quantile normalized FPKM) Z scores of selected inflammation related genes. Scores of individual samples are overlaid on plots as points. (C) Heatmap of gene expression of $50 \mathrm{DE}$ genes with the highest significance in the IPA Inflammatory Response and Arrythmia Disease (D) categories. 

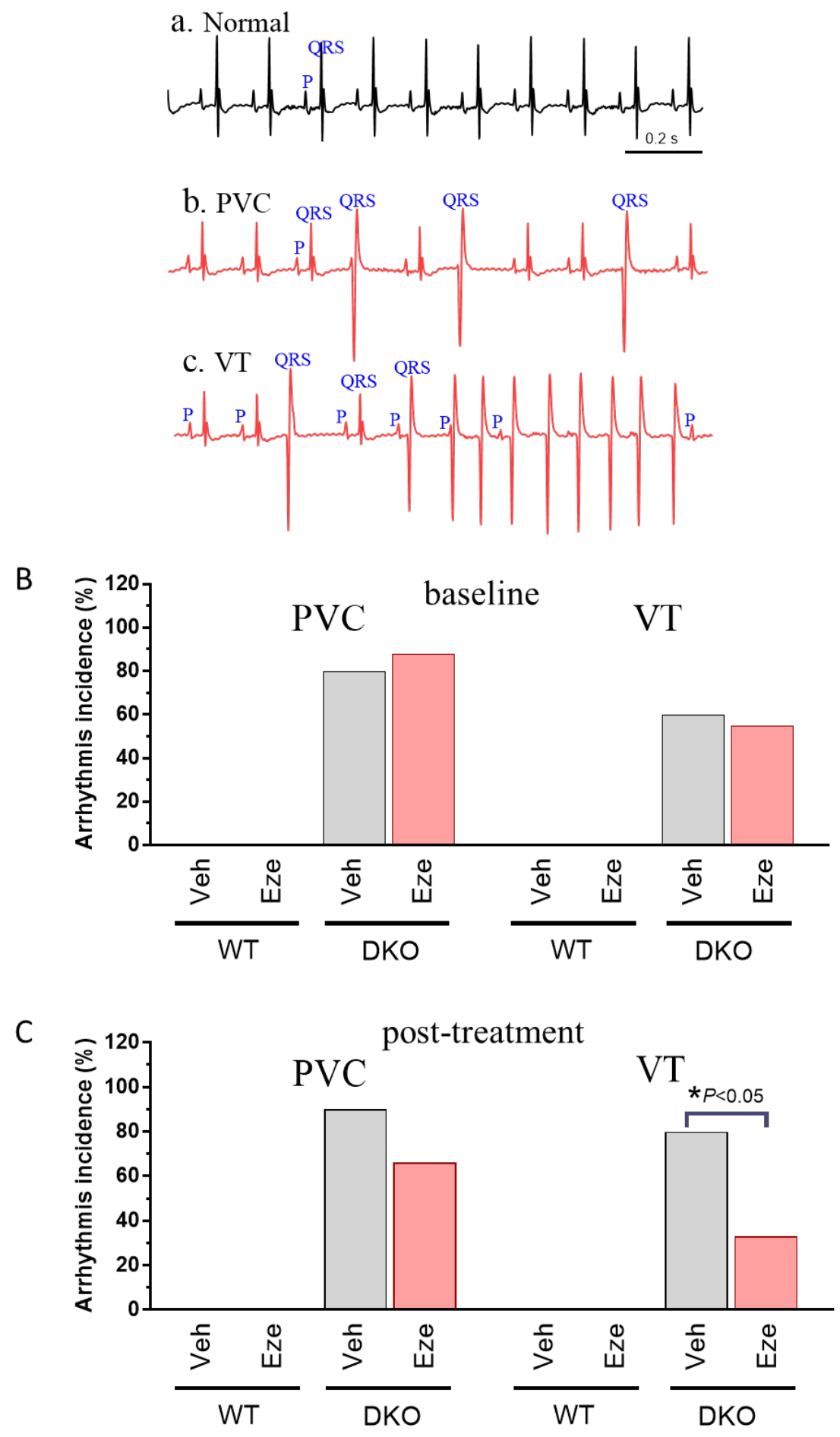

Fig. 5. Ezetimibe reduces incidence of spontaneous ventricular arrhythmias in ABCG5/8

DKO mice. (A) Spontaneous ventricular arrhythmias in ABCG5/8 DKO mice. $\boldsymbol{a}$. normal sinus 
rhythm; $\boldsymbol{b}$, premature ventricular contraction (PVC); $\boldsymbol{c}$, ventricular tachycardia (VT). (B) Incidence of ventricular arrhythmias at baseline (upper panel) and after vehicle and ezetimibe treatment (lower panel).

Fig. 6

个irculating phytosterols
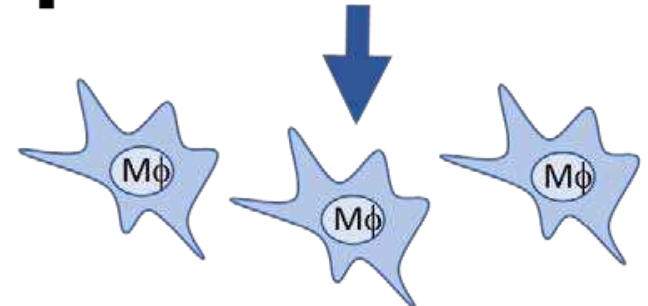

$\mathbf{T}_{\text {cytokines }}^{\text {pro-inflammatory }}$
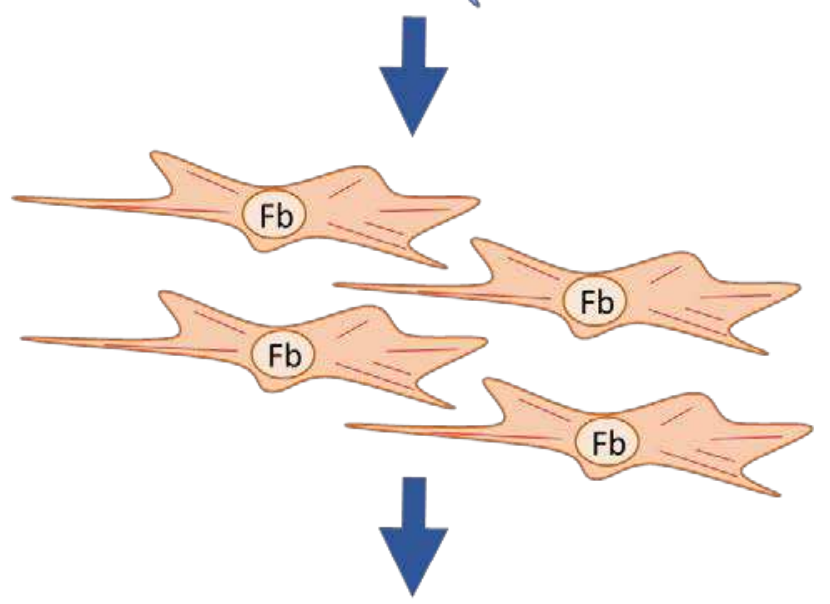

cardiac fibroblast activation, proliferation, fibrosis

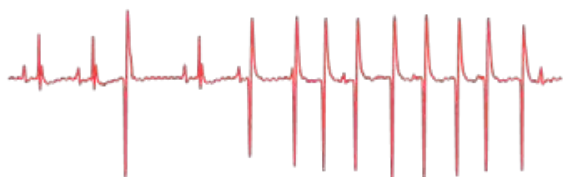

arrhythmias

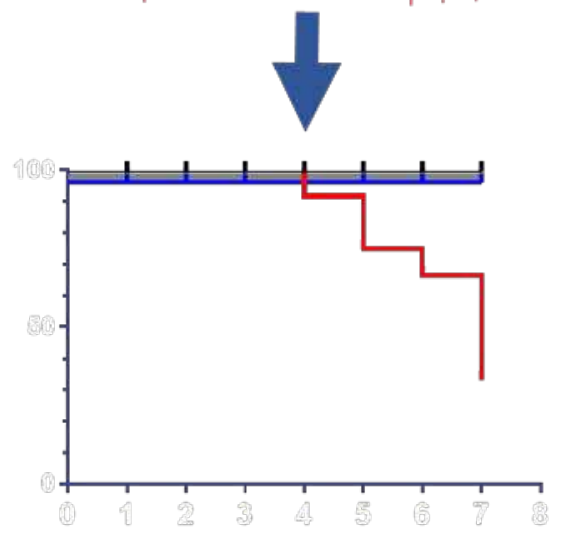

death 
Fig. 6. Our data support a model where elevated circulating phytosterols trigger inflammation, leading to fibroblast activation that cause structural rearrangements to the heart causing cardiac injury, arrhythmia and death. 


\section{Figures}
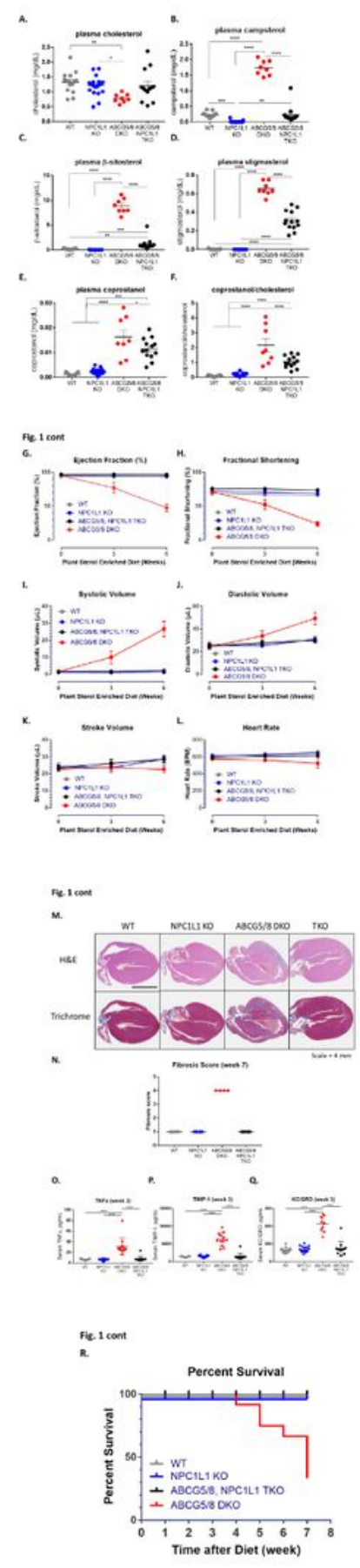

\section{Figure 1}

The NPC1L1 / ABCG5/8 TKO exhibits less phytosterolemia relative to ABCG5/8 DKO littermates. The ABCG5/8 DKO led to a decrease of plasma cholesterol (A) and increase of four phytosterols (campsterol (B), $\beta$-sitosterol (C), stigmasterol (D), coprostanol (E)) as well as the coprostanol/cholesterol ratio (F). 
Less but still significant levels of phytosterols accumulated in the TKO mice in comparison to the mouse cohorts carrying functional ABCG5/8 transporter. DKO - ABCG5/8 double knockout, TKO - ABCG5/8, NPC1L1 triple knockout. Individual data points (circles) are shown together with the sample mean (bar) \pm SEM. ABCG5/8 DKO mice exhibit decreased ejection fraction (G) and fractional shortening $(H)$ and an increase in both systolic $(\mathrm{I})$ and diastolic volume $(\mathrm{J})$ with negligible changes to stroke volume $(\mathrm{K})$ or heart rate $(L)$ indicative of impaired cardiac function and left ventricular dilation. These impairments in cardiac function are not observed for the TKO, NPC1L1 KO or WT littermate controls. DKO - ABCG5/8 double knockout, TKO - ABCG5/8, NPC1L1 triple knockout. Data represented as group means (circles) \pm SEM. WT: $N=15 /$ group; NPC1L1 KO: $N=20-21$ / group; TKO: $N=11-13$ / group; DKO: N = 6-12 /group. Data analyses performed blinded. (M) Representative images from WT, NPC1L1 KO, ABCG5/8 DKO and NPC1L1 / ABCG5/8 TKO at 7 weeks following the switch to the phytosterol enriched diet are shown. Scale bar equal $4 \mathrm{~mm}$. (N) Cardiac fibrosis was scored as 0-within normal limits, 1-minimal, 2-mild, 3moderate, and 4-severe. Severe fibrosis in the ABCG5/8 DKO group at week 7 was associated with a significant increase in several inflammatory markers (serum TNF- $a(0)$, TIMP-1 (P) and KC/GRO (Q)) at week 3. DKO - ABCG5/8 double knockout, TKO - ABCG5/8, NPC1L1 triple knockout. Individual data points (circles) \pm SEM are shown. Significance measured by one-way ANOVA - Tukey post-test (NS - not significant; * $\mathrm{P}<0.05$; ** $\mathrm{P}<0.01$; $* \star \star \mathrm{P}<0.001$; $* \star \star \star ~ P<0.0001)$. (R) ABCG5/8 DKO mice died beginning 4 weeks post-initiation of the phytosterol enriched diet. In contrast, the phytosterol enriched diet did not result in death in the TKO, NPC1L1 KO or WT littermate controls out to week 7 post-diet induction. DKO ABCG5/8 double knockout, TKO - ABCG5/8, NPC1L1 triple knockout. WT: N = 15/ group; NPC1L1 KO: N = 21 / group; TKO: $\mathrm{N}=13$ / group; DKO: $\mathrm{N}=12$ /group when mice were switched to the phytosterol enriched diet. 
A.

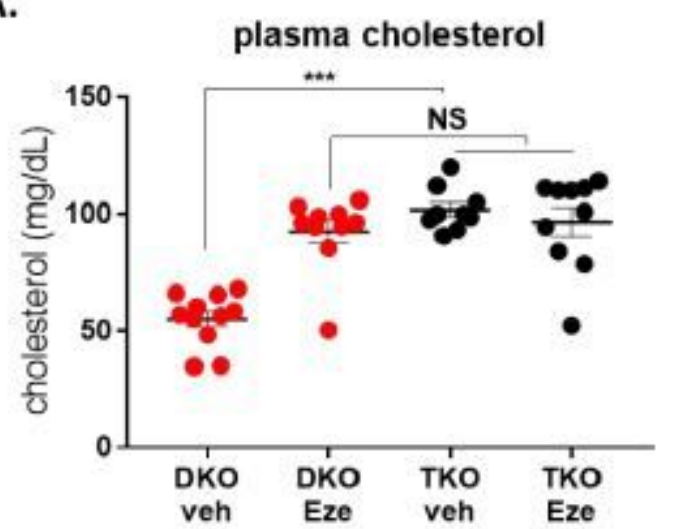

C.

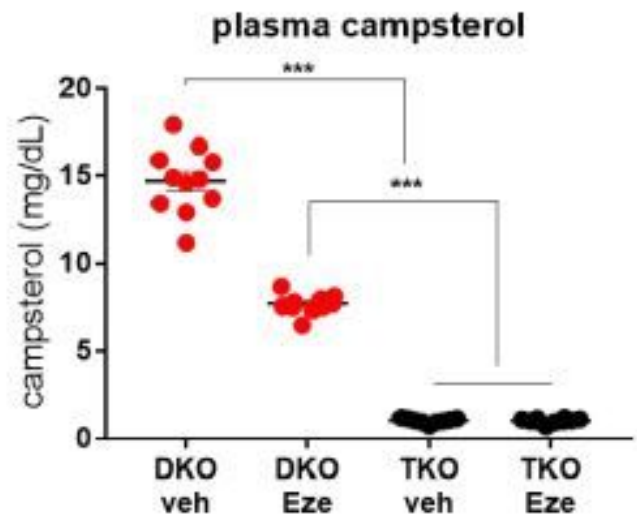

E.

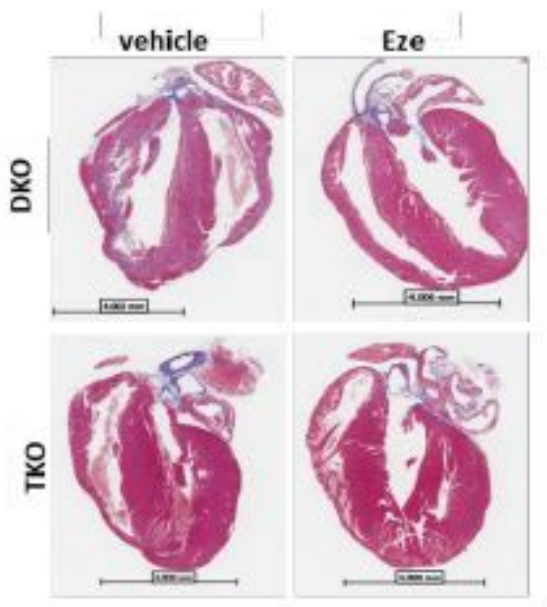

B.

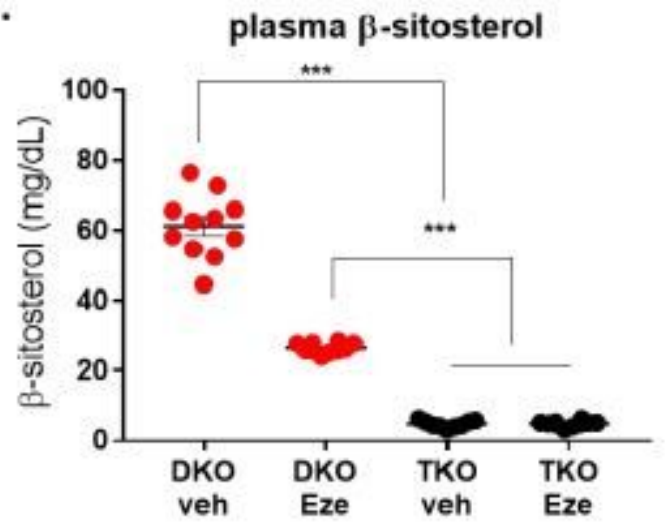

D.

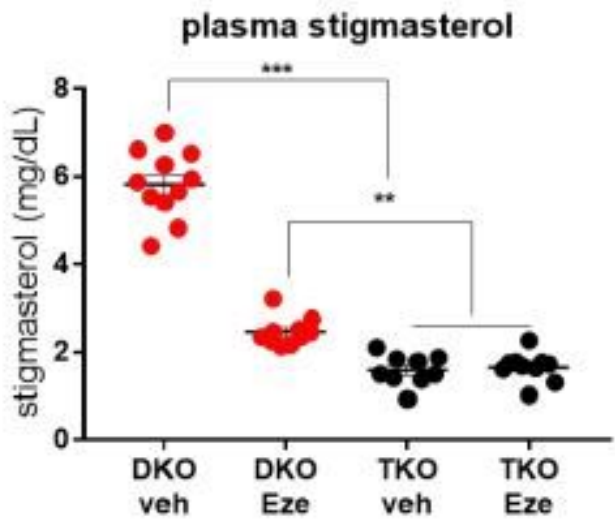

F.

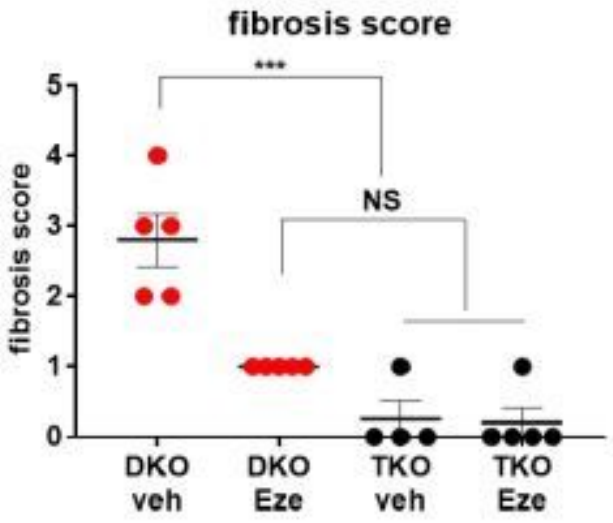

\section{Figure 2}

The NPC1L1 inhibitor, ezetimibe, partially prevents ABCG5/8 deficiency-derived phytosterolemia.

Ezetimibe treatment led to an increase of plasma cholesterol (A) and decreased of three phytosterols ( $\beta$ sitosterol (B) campesterol (C) and stigmasterol (D)) in ABCG5/8 DKO mice. Plasma levels of phytosterols were lower in the NPC1L1 / ABCG5/8 TKO relative to the ABCG5/8 DKO littermates treated with ezetimibe. Both ezetimibe and NPC1L1 KO decreased ABCG5/8 DKO induce fibrosis (E, F). DKO ABCG5/8 double knockout, TKO - ABCG5/8, NPC1L1 triple knockout, veh - vehicle, Eze - ezetimibe. 
Individual data points (circles) are shown together with the sample mean (bar) \pm SEM. Significance measured by one-way ANOVA - Tukey post-test (NS - not significant; ${ }^{\star} \mathrm{P}<0.05$; $* \star \mathrm{P}<0.01$; $* \star \star \mathrm{P}<0.001$; $\star \star \star \star ~ P<0.0001)$. Histo-pathological assessment performed blinded.

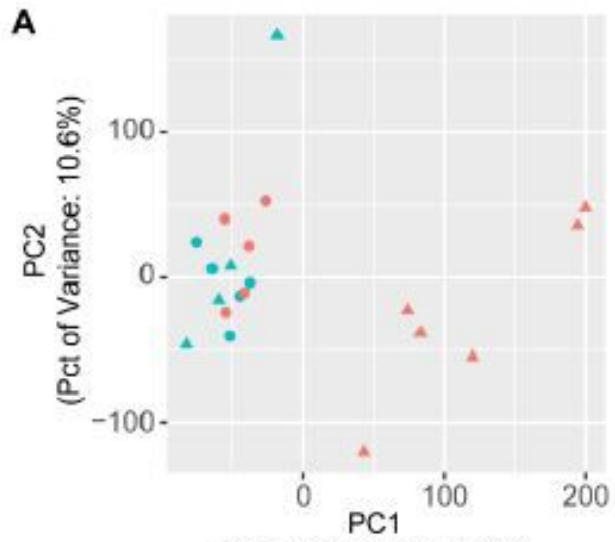

(Pct of Variance: $25.6 \%$ )

Genotype + Treatment

- ABCG5/8 DKO

- ABCG5/8 DKO + Ezetimibe

- ABCG5/8 NPC1L1 TKO

- ABCG5/8 NPC1L1 TKO + Ezetimibe

C

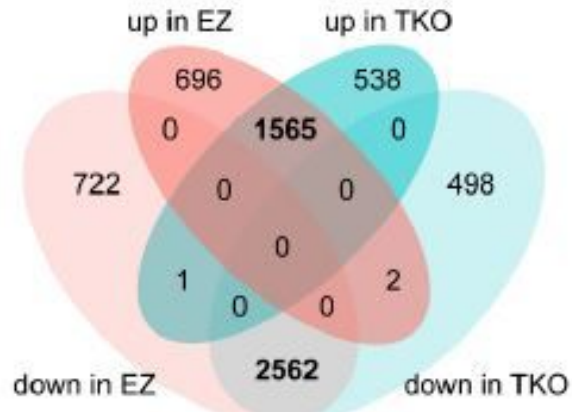

B Differential Gene Expression

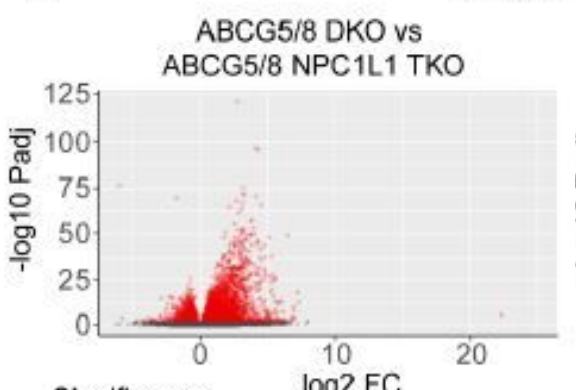

Significance

- not significant

- Padj $<0.01$

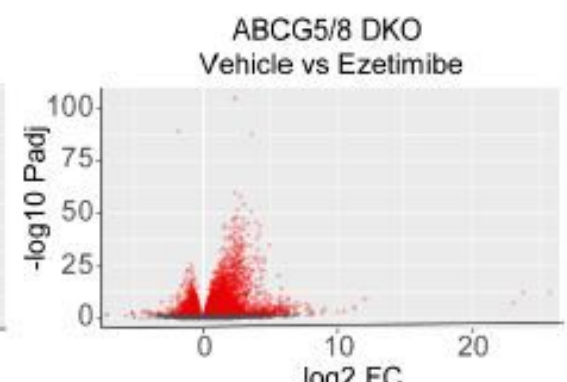

$\log 2$ FC

D

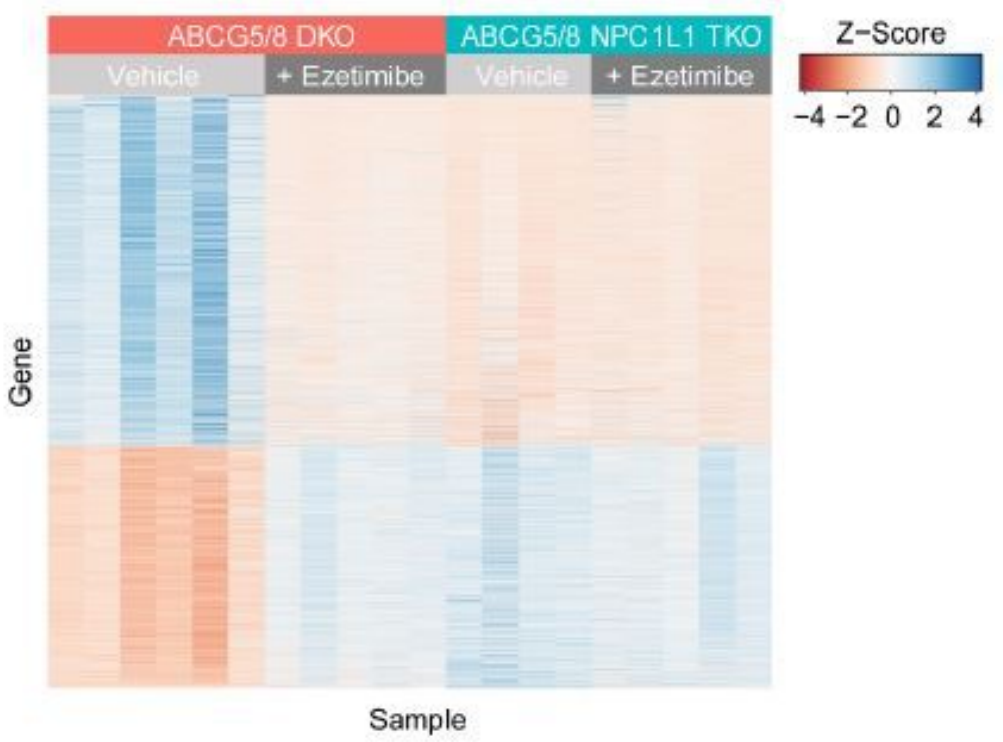

Figure 3

RNA-seq analysis of heart samples. (A) Plot of first two components of principal component analysis (PCA) of gene expression. ABCG5/8 double knock-out (DKO) samples are filled with red and ABCG5/8 NPC1L1 triple knock-out (TKO) samples are filled with cyan. Vehicle samples are triangles and ezetimibe treated samples are circles. (B) Volcano plots of $p$ values from differential expression analysis versus log2 fold change in gene expression as calculated using DEseq2 comparing DKO untreated to TKO or DKO ezetimibe treated samples. A positive fold change value indicates higher expression in ABCG5/8 DKO untreated samples. (C) Venn diagram demonstrating overlap in differentially expressed (DE) genes from two comparisons. (D) Heatmap of gene expression (quantile normalized FPKM) Z-scores of DE genes identified comparing DKO and TKO conditions. 


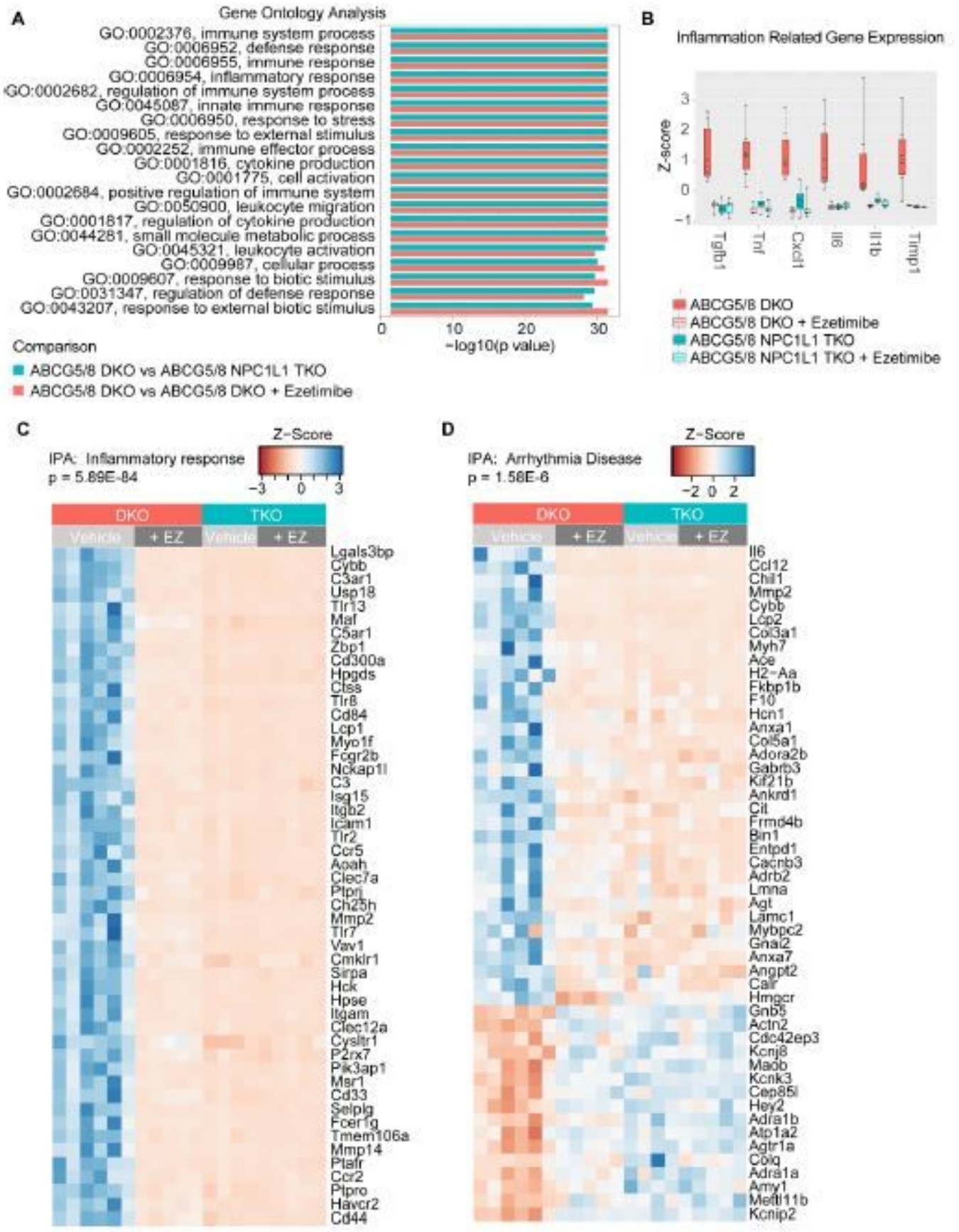

Figure 4

Functional analysis of RNA-seq data. (A) Gene Ontology (GO) enrichment analysis of differentially expressed (DE) genes comparing ABCG5/8 double knock-out (DKO) samples to ABCG5/8 NPC1L1 triple knock-out (TKO) (cyan) or DKO ezetimibe (red) treated samples. P values for the top twenty GO categories from the DKO/TKO comparison are plotted. (B) Box plots of gene expression (quantile normalized FPKM) Z scores of selected inflammation related genes. Scores of individual samples are overlaid on plots as points. (C) Heatmap of gene expression of $50 \mathrm{DE}$ genes with the highest significance in the IPA Inflammatory Response and Arrythmia Disease (D) categories. 

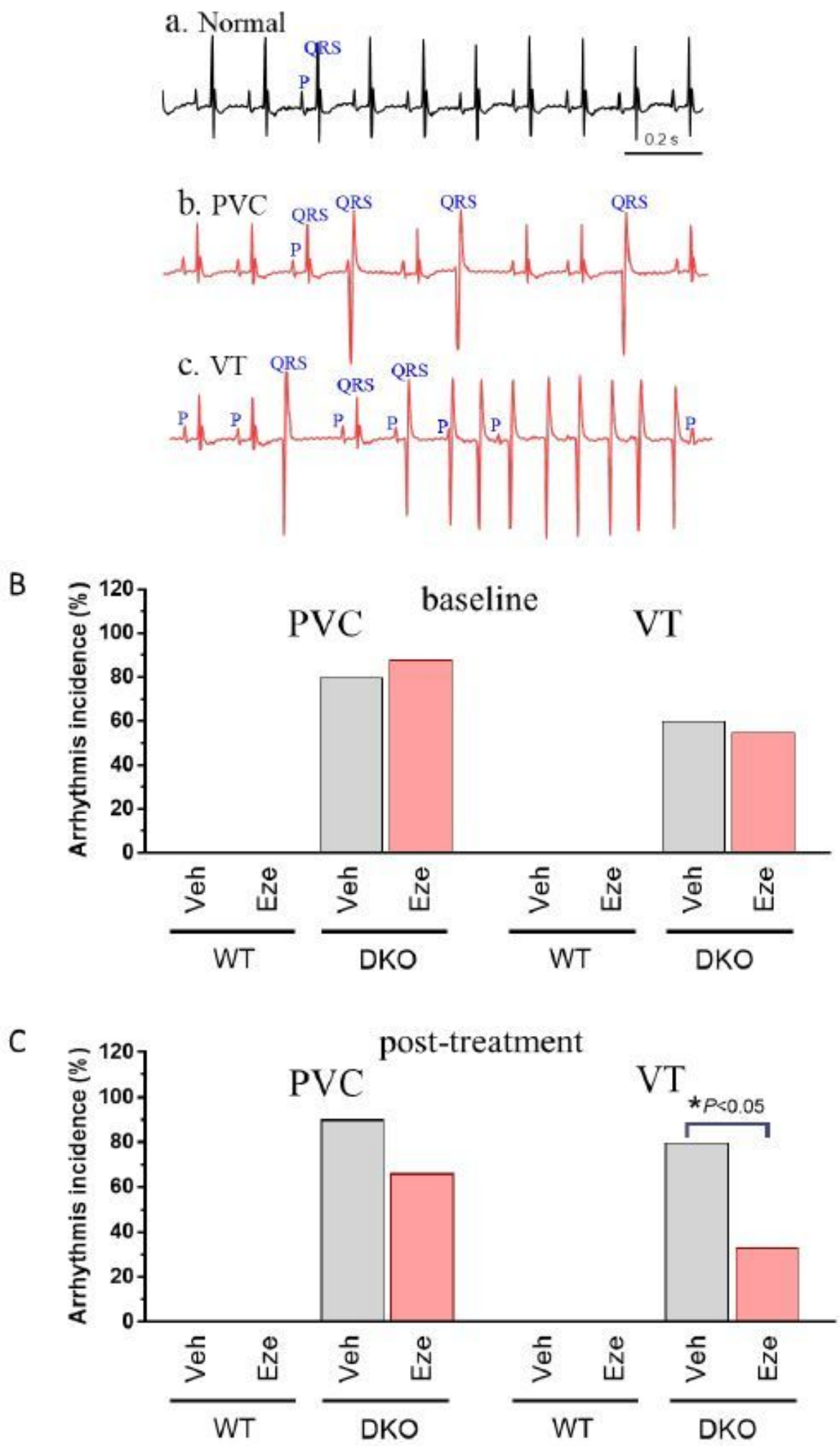

Figure 5

etimibe reduces incidence of spontaneous ventricular arrhythmias in ABCG5/8 DKO mice. (A) Spontaneous ventricular arrhythmias in ABCG5/8 DKO mice. a. normal sinus rhythm; $b$, premature ventricular contraction (PVC); c, ventricular tachycardia (VT). (B) Incidence of ventricular arrhythmias at baseline (upper panel) and after vehicle and ezetimibe treatment (lower panel). 
Fig. 6 circulating phytosterols

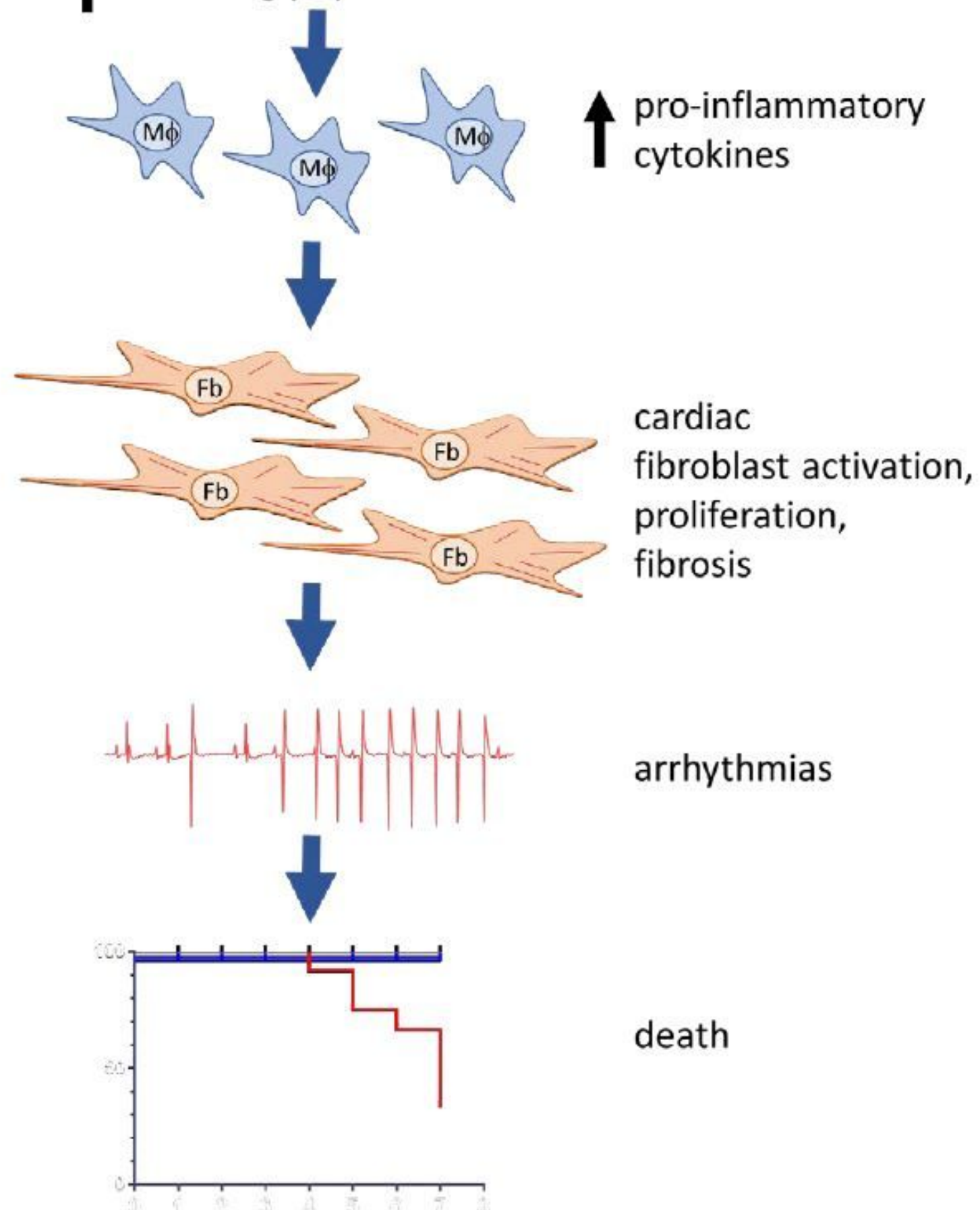

Figure 6

Our data support a model where elevated circulating phytosterols trigger inflammation, leading to fibroblast activation that cause structural rearrangements to the heart causing cardiac injury, arrhythmia and death. 
This is a list of supplementary files associated with this preprint. Click to download.

- Supplement.pdf

- supplementaltable1.xIsx 